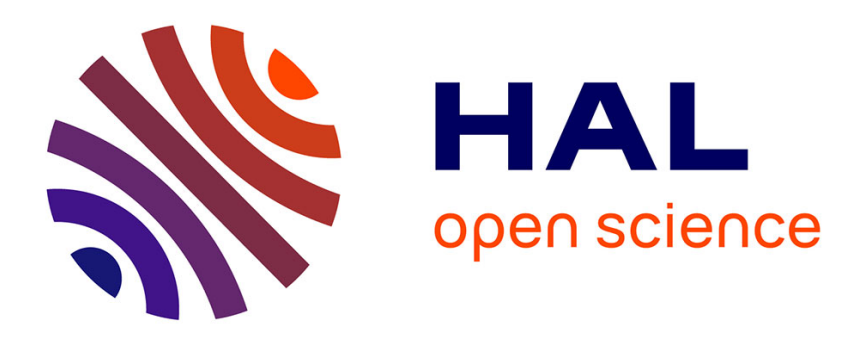

\title{
La science du langage en France de 1870 à 1885: du marché civil au marché étatique
}

Gabriel Bergounioux

\section{To cite this version:}

Gabriel Bergounioux. La science du langage en France de 1870 à 1885: du marché civil au marché étatique. Langue française, 1984, 63 (1), pp.7 - 41. 10.3406/lfr.1984.5195 . halshs-01820544

\section{HAL Id: halshs-01820544 https://shs.hal.science/halshs-01820544}

Submitted on 21 Jun 2018

HAL is a multi-disciplinary open access archive for the deposit and dissemination of scientific research documents, whether they are published or not. The documents may come from teaching and research institutions in France or abroad, or from public or private research centers.
L'archive ouverte pluridisciplinaire HAL, est destinée au dépôt et à la diffusion de documents scientifiques de niveau recherche, publiés ou non, émanant des établissements d'enseignement et de recherche français ou étrangers, des laboratoires publics ou privés. 


\section{La science du langage en France de 1870 à 1885 : du marché} civil au marché étatique

In: Langue française. $\mathrm{N}^{\circ} 63,1984$. pp. 7-41.

Citer ce document / Cite this document :

Bergounioux Gabriel. La science du langage en France de 1870 à 1885 : du marché civil au marché étatique. In: Langue française. $\mathrm{N}^{\circ} 63,1984$. pp. 7-41.

doi : $10.3406 /$ fr. 1984.5195

http://www.persee.fr/web/revues/home/prescript/article/lfr_0023-8368_1984_num_63_1_5195 


\section{LA SGIENCE DU LANGAGE EN FRANCE DE 1870 A 1885 : DU MARCHÉ CIVIL AU MARCHÉ ÉTATIQUE}

A la fin du XIX siècle, en France, la généralisation de l'alphabétisation et la " francisation ", à travers la mise en place de l'école primaire entre autres, dessinent une certaine coïncidence entre les décisions de l'Académie française (et du marché de la linguistique) et les productions du marché linguistique '. Qu'il s'agisse de l'orthographe (celle de l'Académie française devient officielle en 1832) ou du refoulement du patois hors des situations contraintes, la norme est imposée pratiquement à un nombre croissant de locuteurs confrontés simultanément à leur inclusion linguistique dans le marché linguistique et à leur exclusion sociale par le marché de la linguistique.

Le marché de la linguistique semble assuré d'un certain équilibre : écoles littéraires et "gens de lettres" (journalistes, publicistes, prédicateurs, historiens...) se partagent une autorité sur la langue que ne discutent pas ceux qui se prévalent d'une compétence, les grammairiens (Girault-Duvivier, Noël, Larive, Landais...), les lexicographes (Nodier, Guizot, Laveaux, Roquefort, Bescherelle, Littré...), les philosophes spécialistes de l'ancien français (Paulin Paris, Francisque Michel, Génin, Quicherat...). Ceux qui font métier de professer des langues autres que le français sont exclus de tout pouvoir sur le marché linguistique.

Avant 1870, le marché de la linguistique semble à la fois anomique et uniforme par rapport à l'ensemble du champ de production des biens symboliques, socialement, épistémologiquement et chronologiquement. Les savoirs s'épuisent dans un travail indéfiniment reconduit de compilation, qu'il s'agisse des Amusements philologiques (1825) de G. Peignot ou des dictionnaires ${ }^{2}$, sans parler de la Grammaire des grammaires (1812)

1. Le marché linguistique est constitué par l'ensemble des locuteurs d'une langue à travers leurs échanges linguistiques dans cette langue. Le marché de la linguistique comprend les seules personnes liées à la production de théories et de jugements sur l'ensemble des langues et qui cherchent à se faire conférer sur ces questions une autorité qui dépasse leurs simples capacités de locuteur du marché linguistique.

2. Dans son Histoire des Dictionnaires français (1968), Georges Matoré a mis en évidence la fallacieuse recherche d'une exhaustivité lexicale au détriment de l'étude sémantique ou étymologique, jusqu'à Larousse et Littré non compris. 
de Girault-Duvivier dont le titre est à prendre au pied de la lettre. L'orientalisme, après les succès des indianistes (Anquetil du Perron et Chézy), sinisants (Langlès), sémitistes (Audran et Sylvestre de Sacy), après les découvertes de Champollion, se rétracte peu à peu : à la mort de Burnouf, en 1852, il ne sera pas possible d'élire un successeur dans sa chaire du Collège de France. Seul le latin, et à un moindre degré le grec, soutenu par une formule scolaire en régression, parvient à garder une relative vitalité.

Afin de restituer la logique opératoire à l'œuvre dans la transformation du marché de la linguistique, nous allons :

1) récapituler sommairement les acquis de la linguistique allemande;

2) reconstruire la situation sociale, institutionnelle et scientifique à cette date en France;

3) analyser la portée de la création de l'École Pratique des Hautes Études (E.P.H.E.), notamment de sa IV e section : "Sciences historiques et philosophiques $"$.

\section{La situation avant $\mathbf{1 8 7 0}$}

\section{Naissance de la linguistique}

Retrouver et pondérer les principes qui conduisirent à une définition scientifique de la linguistique serait long: outre le rôle premier d'un rationalisme en souci de rupture avec les conceptions métaphysiques du langage, l'émergence d'un réel où la science trouve prise à symbolisation tient à deux efforts concomitants : d'abord, la collecte systématique de documents linguistiques ${ }^{3}$ - en France, Sylvestre de Sacy et l'arabe, Anquetil du Perron et le "zend ", Audran et l'hébreu, Langlès et le chinois, Chézy et le sanscrit, Raynouard et l'occitan, Le Gonidec et le breton, Champollion et les hiéroglyphes égyptiens... - ensuite, une approche plus rigoureuse de la forme phonétique des langues. Les savants français disposaient d'une réelle avance au début du XIX ${ }^{e}$ siècle : les découvertes décisives de la Vergleichende Grammatik, la " grammaire comparée " de Bopp, l'anéantissent dès 1816 et l'Allemagne s'arroge une primauté incontestée pendant un demi-siècle. Il ne nous appartient pas de rendre compte de cette découverte qui supposerait que soient reconstruites les trajectoires du groupe d'étudiants et de professeurs allemands (les frères Schlegel, Bopp, Humboldt) qui poursuit des études à Paris de 1805 à 1815 et l'organisation du marché allemand de la philologie sur lequel s'impose assez rapidement Bopp.

La révolution copernicienne de Bopp fut de poser comme une fin ce qui était considéré comme un moyen : la langue des textes étudiés par les philologues; pour reprendre sa formule : "La langue en elle-même

3. Qui agit comme une révision de toute théorie de * la langue *, soit originelle (l'hẻbreu), soit parfaite (le français), et permet de mettre en jeu une théorie de la différence fondée sur la comparaison. C'est le principe d'un des postulats fondamentaux de la linguistique scientifique, sans cesse à reconquérir sur le sens commun: que toutes les langues se valent. 
et pour elle-même. * Rompre la perception globale qui confondait la langue et la littérature ou la religion pour s'attacher à la langue seule supposait une objectivation désacralisante, une autonomie artificiellement constituée pour l'observation et où le sujet n'avait plus prise.

Quelle procédure d'observation permit la théorie de la grammaire comparée? D'abord, le parallèle établi entre plusieurs langues au lieu d'un effort exclusif portant sur un seul idiome ${ }^{4}$. Au rebours de toute interrogation esthétique ou métaphysique ${ }^{5}$, les textes sont décomposés, décontextualisés en listes paradigmatiques dans lesquelles la morphologie - déclinaisons et conjugaisons - prime la syntaxe et la lexicologie. Cette méthodologie donne son nom à la " grammaire comparée " dont la désignation sera source de malentendus ${ }^{6}$. La réinsertion des paradigmes dans la forme conventionnelle des grammaires (ordre des personnes et des cas, séparation typographique des affixes et des radicaux, disposition en colonnes) oriente la lecture vers un ensemble réglé de concordances et de variations.

La rupture première de l'objectivation est dans cette fracture qui se dessine entre le texte saisi dans sa solidarité syntagmatique et l'image d'une langue établie paradigmatiquement selon les procédés ordinaires puis rapprochée d'autres langues mises en liste selon les mêmes catégories (le présent répondant au prêsent, le duel au duel, l'instrumental à l'instrumental, le neutre au neutre...) Le "désenchantement du monde* caractéristique de toute avancée scientifique se lit dans cette philologie méticuleuse si opposée à la vénération des textes, si désacralisante puisqu'elle établit sur le même plan des langues sans renom ni littérature - le gotique, le lituanien - et des langues socialement valorisées comme le latin, le grec ou le sanscrit.

Pour que la comparaison prenne sens, elle devait porter sur des formes phonétiques et ce fut une des chances de Bopp de travailler sur des langues dont l'orthographe - ou la transcription - était conforme, dans sa convention, à la prononciation; aussi, dans ses premiers travaux, Bopp parle de rapprocher des "lettres" et non des sons. Les progrès de la phonétique et l'étude de langues à orthographe complexe - français, anglais - transcrites en caractères latins requéraient une notation uniforme des sons, une écriture propre à cette science nouvelle : la linguistique.

Trois solutions concoururent entre les linguistes : la première utilisa la notation des sons réalisée dans l'orthographe du pays, système de transcription vernaculaire dans sa conception (ce qui freinait sa diffusion internationale) et son application (il fallait le compléter pour les phonèmes des

4. La comparaison entre plusieurs langues, déjà présente au xvie siècle, avait été développée par les celtomanes et Raynouard.

5. Interrogations qui feront retour, de n'avoir pas été analysées, dans la vision de l'évolution linguistique (Bopp: - Je travaille dans un champ de ruines $\rightsquigarrow$ ) et des qualités intrinsèques des langues.

6. Les philologues francais insistent, dans la grammaire, sur l'axe syntagmatique (ordre des mots, règles d'accord) plutôt que sur l'axe paradigmatique d'où des équivoques sur le sens de "grammaire comparée * dans les débuts de la discipline. 
autres langues $\left.{ }^{7}\right)$. La seconde, inspirée semble-t-il des procédés sténographiques qui visent la restitution du son, recourut à une suite de signes conventionnels ${ }^{8}$. La troisième solution fut l'établissement d'un compromis entre les différentes correspondances son/lettre de l'alphabet romain telles qu'elles existaient entre les pays dont les membres appartenaient à l'Association phonétique internationale. Pour que la linguistique dispose de son écriture, il fallut préalablement une instance supranationale qui garantisse la pertinence et la validité de cette notation perçue comme concurrente de l'orthographe reçue des différentes langues ?

L'alphabet phonétique international (A.P.I. en abréviation) reflète assez bien l'état du marché mondial de la linguistique à la fín du XIXe siècle. A l'exception de quelques Russes, tous les linguistes utilisent l'alphabet latin - et l'enchaînement de l'écriture de gauche à droite - dont ils font l'armature de l'A.P.I. accordant ainsi le primat à la composante articulatoire au dépens des phénomènes de ton ou de longueur.

La " méthode comparée", pour reprendre la dénomination de Meillet, semble heuristique à proportion de la transparence de son objet, les langues. Celles-ci doivent être objectivées, " pensées comme des choses ", c'est-à-dire séparées des sujets parlants. Il semble que cette procédure ait été facilitée de s'exercer sur des langues mortes mais que cette facilité ait contribué au philologisme qui, refoulant la dimension sociale de la langue, écartait le versant sémantique et l'analyse du discours pour s'en tenir à une combinatoire de formes, un jeu formel. La méthode comparée se donne pour fin la répétition d'une variation à travers des unités de même niveau collectées à des moments différents afin d'en déduire une loi de correspondance. Deux paramètres apparaissent cruciaux : l'un de l'ordre du langage dans ce qu'en saisit son organisation paradigmatique, l'autre de l'ordre du temps réinterprété en termes d'histoire, de civilisation ou de race. Si, en 1816, Bopp assigne l'origine des langues qu'il étudie au sanscrit, vers 1850, Schleicher impose l'indo-européen reconstruit.

L'heuristique comparatiste est au principe de grammaires historiques consacrées à d'autres familles - les langues chamito-sémitiques (1850-1890), le nippo-coréen (1907) - ou à des groupes de la famille indo-européenne - langues romanes $(1836)^{10}$, langues celtiques (1851) ${ }^{11}$. Le développement scientifique du marché de la linguistique allemand, probablement lié à la forte croissance universitaire et scientifique d'outreRhin, ne semble guère être perçu en France où tout semble s'opposer à

7. Système le plus évident, le plus proche du sens commun, il est appliqué au provençal par Mistral dès 1856 et, sous une forme très améliorée, par la Revue des Patois jallo-romains de Rousselot et Gilliéron (1887).

8. Ces signes formaient un alphabet dit organique restituant les points d'articulations; système utilisé par Bell et Sweet.

9. En reprenant l'opposition médiévale nomen (" gé $*) / f i g u r a(* g, G *) /$ potestas $(/ g /, z /)$, on peut dire que l'A.P.I. unifiait la potestas (un seul son par lettre), divisait la figura $(\mathrm{g} \neq \mathrm{G}$ ) et supprimait le nomen (identique à la potestas). Paul Passy, qui fut parmi les fondateurs de l'A.P.I., présente la transcription du francais en 1886.

10. Diez, Vergleichende Grammatik der romanischen Sprachen (1836), Diez est professeur à l'Université de Bonn.

11. Zeuss, Grammatica celtica (1851); Zeuss n'a pas obtenu, à cette date, de poste dans l'enseignement supérieur. Son ouvrage paraît en latin. 
une redéfinition homologue : une tradition philosophique différente, un enseignement supérieur mal structuré, la résistance des puristes et des mondains, l'ignorance de l'allemand et le manque d'intérêt pour les langues étrangères, le poids de la tradition rhétorique en lettres et en histoire, le mépris pour la recherche germanique...

\section{Les postes du marché de la linguistique en France}

Une des raisons invoquées pour justifier le désintérêt des grammairiens français, leur refus du comparatisme allemand, est l'absence de lieu où un tel enseignement eût pu être repris. C'est déjà ce que supposait Hippolyte Fortoul ${ }^{12}$ lorsqu'il prévoyait de programmer, dans les lycées et les facultés, des cours d'histoire de la langue française afin de restituer une continuité entre les humanités - le latin et le grec - et la littérature française. Un volant de postes est préparé, notamment par des missions de recherches archéologiques pour le Corpus Inscriptionum de la Gaule et de l'Algérie et ethnographiques pour le Recueil des Chants populaires du Midi. Au-delà des nécessités scientifiques, la langue rétablie dans sa durée devait retracer l'organisation des thèmes d'un nationalisme nouveau, conjoignant les thèmes conservateurs de la patrie et de son histoire, de la celtitude ${ }^{13}$ et de la romanité, de la terre et de la religion, et les connaissances d'outre-Rhin. Cet hommage au sol et à la civilisation rendu au travers de la langue enseignée à l'école relève plus d'un technocratisme des idées que $d$ 'un scientisme. On se gardait d'enfreindre les respects établis: la langue ne se relativisait qu'en regard de sa propre histoire et rien des accaparements puristes n'en devait être ébranlé. La mort de Fortoul a clos l'œuvre entreprise et non reprise après la disparition de son promoteur.

Néanmoins, quatre institutions auraient pu recueillir les leçons de la grammaire historique : le Collège de France, l'École des Chartes, l'École nationale des Langues orientales vivantes et les facultés, particulièrement la Sorbonne.

Au Collège de France, quatre chaires sont concernées, dont deux, de création récente - elles n'ont pas quarante ans -, portent dans leur intitulé les marques de l'esprit romantique qui les inspira :

- "Langue et littérature du Moyen Age ", occupée par Paulin Paris ${ }^{14}$; - "Langues et littératures du Midi de l'Europe", occupée par Edgar Quinet ${ }^{15}$.

Deux chaires plus anciennes ont leur importance :

12. H. Fortoul (1811-1856), professeur de faculté originaire de la Provence, sensible aux thèses romantique et germanophile; devient le premier ministre de l'Instruction publique de l'Empire autoritaire. Gaules.

13. Ce fut explicitement à Napoléon IIl que revint la paternité du mythe de Vercingétorix, roi des

14. Paulin Paris (1800-1881), étudiant en droit, il est attaché à la Bibliothèque royale; autodidacte en ancien francais, philologue, son élection au Collège de France a lieu en 1853 .

15. Edgar Quinet (1803-1875), plutot philosophe que philologue, Quinet consacra son enseignement à une réflexion sur la civilisation et la littérature. 
- "Langue et civilisation de l'Inde ancienne ", que Burnouf garda jusqu'à sa mort, en 1852, et pour laquelle nul successeur ne put être trouvé; - "Poésie latine", sous un intitulé très classique, Ernest Havet orienta son enseignement vers la métrique et l'étude de la langue.

A l'exception de Burnouf, aucun de ces professeurs ne semble avoir eu connaissance, scientifiquement, de la grammaire comparée. Encore Burnouf ne s'en fit-il pas le propagandiste ${ }^{16}$. L'absence de sélection de l'assistance, la distance conservée face aux élèves, le principe même de la conférence appauvrissaient les possibilités d'une élaboration scientifique des contenus; l'absence de diplômes, de débouchés décourageait les étudiants. L'attente du public comme la formation des maîtres jouait contre la spécialisation et l'objectivation épistémologiquement nécessaires.

A l'École des Chartes (fondée en 1821), un poste de professeur est réservé à l'enseignement de l'ancien français, occupé par Francis Guessard (1814-1882) depuis 1854 sous l'intitulé : "philologie romane". $\mathrm{Ne}$ connaissant pas l'allemand, Guessard partage la prévention générale qui sévit à l'encontre de la grammaire historique. Ni Anatole de Montaiglon ${ }^{17}$, ni Léon Gautier ${ }^{18}$, tous deux éditeurs de manuscrits et spécialistes du Moyen Age, ne s'initièrent vraiment à la grammaire historique. Jules Quicherat, le directeur des Chartes, reste un spécialiste de la toponymie. Dans tous ces projets, aucun ne s'attachait à la langue en elle-même et pour elle-même.

Quant aux facultés, elles représentent un profond conservatisme scientifique, politique et religieux : proches de l'enseignement secondaire par leurs programmes et leur public, elles restent étrangères au nouvel horizon scientifique qui se dégage. Une seule exception: Émile Egger (1813-1885), auteur de Notions élémentaires de grammaire comparée des langues classiques en 1852, bon connaisseur des travaux allemands, obtient la chaire de littérature grecque de la Sorbonne en remplacement de Boissonnade en 1855 mais n'en fait pas un centre de rayonnement des connaissances nouvelles. Au-delà du poste, il manque l'univers de croyance dans une rationalité présidant aux faits de langue et l'appareil de revues, d'édition ou de sociétés qui donnerait un sens au savoir que peut mobiliser Egger. Professeur à l'E.N.S., Egger se situe en conformité avec la demande scolaire et universitaire, induit, faute d'élèves, de pairs, ou seulement d'une demande, à se conformer à l'image reçue du professeur de grec.

Quant à l'École nationale des Langues orientales vivantes (dont l'origine remonte au XVII siècle et l'organisation à la fin du XVIII'), elle est confinée dans l'apprentissage des langues à des fins pratiques et semble avoir renoncé à la recherche. Quelques chercheurs interfèrent sur le marché de la linguistique sans disposer d'un capital scientifique suffisant pour le modifier, soit par méconnaissance du marché allemand - tel

16. Mais la conscience de la supériorité de la grammaire comparée devait déjà exister si l’on considère le cas de Gaston Paris et de Louis Havet.

17. A. de Montaiglon (1824-1895), chartiste et philologue, professeur de bibliographie à l’École des Chartes. phie.

18. L. Gautier (1832-1897) : chartiste, professeur de poésie latine, de diplomatique et de paléogra- 
Génin ${ }^{19}$-, soit par spécialisation dans l'édition de textes ou la bibliographie - tels Delisle ou Guérard, tous deux conservateurs de la Bibliothèque nationale -, soit, tel Littré, parce qu'ils usaient de la linguistique comme d'un moyen et non comme d'une fin, en marge de l'enseignement supérieur.

Vers 1865-1870, il n'y a pas de marché de la linguistique ${ }^{20}$ en France en dehors de celui engendré par les contraintes du marché linguistique (national et international). Aucun savoir, au croisement d'une écriture, d'une méthode et d'une théorie, comme en Allemagne, ne paraît assurer au champ une hiérarchisation ou une logique surimposant son fonctionnement propre à l'encontre des exigences du marché des biens symboliques en entier. L'enseignement du latin et du grec reste tributaire de part en part du système scolaire et la carrière de Génin, ou de Quinet, en se déroulant dans le journalisme ou le commerce des idées, en occupant des positions fortes dans la hiérarchie des avis autorisés sur la langue française et les manières de l'étudier, traduit la continuité sociale et " scientifique " qui préside à la jonction du marché de la linguistique et du marché intellectuel. Nulle part ne se dessine de fracture provoquée par l'ésotérisme scientifique, assurant la redéfinition des frontières en abandonnant la séparation entre ce qui se dit et ce qui ne se dit pas travail du puriste ou du grammairien scolaire - au profit d'une séparation entre ce qui se dit et ce qui ne se dit pas à propos de ce qui se dit, c'està-dire entre discours scientifique et non scientifique, le premier acte d'objectivation consistant à accepter, en théorie, toute production linguistique effectivement réalisée.

D'être en continuité du marché des biens symboliques, le marché de la linguistique est extraverti : sa dépendance méthodologique, institutionnelle et sociale transparaît dans l'inexistence des postes universitaires, l'absence de revues spécialisées (Génin publie ses études dans le journal l'Illustration), la publication de recueils d'anedoctes ou de " curiosités" en lieu et place des manuels et des ouvrages de recherche. Une autre preuve en pourrait être trouvée dans l'absence de terminologie spécifique en sus des termes reçus depuis le XVII et le XVIII siècles. Il semblerait que le marché de la linguistique soit un sous-ensemble du marché littéraire, probablement un des moindres, en marge de la critique et de la philologie. Il fallait la mobilisation des connaissances de la grammaire comparée allemande pour imposer l'autonomie du marché de la linguistique : c'est à quoi allait s'appliquer un groupe très restreint de romanistes et de comparatistes.

\section{La création de l'E.P.H.E.}

A la fin des années 1860 , le retard pris par l'Université française inquiète certains hauts fonctionnaires et le ministre de l'Instruction

19. Francis Génin (1803-1856), journaliste et administrateur, auteur Des variations du langage français depuis le xIr siècle et des Récréations philologiques, éditeur de textes du XVIF; assez représentatif du * mondain " en philologie.

20. Il faudrait rappeler, pour mémoire, qu'en marge des institutions, de nombreux amateurs, curieux de patois, d’onomastique ou d’étymologie, poursuivent un certain travail à prétention linguistique. 
publique, Victor Duruy. Celui-ci envisage une réforme de l'enseignement supérieur qu'il tente d'appliquer en 1866: devant les résistances corporatistes, il y renonce et esquisse, en 1867, un projet organisé autour de quelques maîtres spécialisés, dispensant leur enseignement à un public restreint, tourné vers la recherche. Convaincu par cette première expérience, il la fixe dans un cadre neuf : l'École pratique des Hautes Études, rassemblant six sections, dont la quatrième prend le titre de : "Sciences historiques et philologiques ".

Le recrutement des professeurs de la IVe section de l'E.P.H.E. se fait parmi les rédacteurs de la Revue critique d'Histoire et de Littérature, fondée en 1866 par Paul Meyer, Gaston Paris, Charles Morel, et Hermann Zotenberg. Très ouverts au marché scientifique allemand, les deux romanistes du groupe - Meyer et G. Paris ont suivi les cours de Diez à Bonn - se distinguent surtout par leur jeunesse : en 1868, G. Paris a vingtneuf ans, P. Meyer en a vingt-huit. Appelés par Duruy pour dispenser un enseignement de philologie romane, ils retrouvent dans l'E.P.H.E. un orientaliste : Michel Bréal, formé à Berlin, et un historien, Gabriel Monod, étudiant à Göttingen. Il n'en faut pas plus à leurs adversaires, universitaires ou " mondains", pour stigmatiser la pensée germanique établie contre la Sorbonne ${ }^{21}$.

Contrairement aux idées reçues, l'ignorance des théories comparatistes semble moins liée à la barrière des langues qu'à l'imperméabilité d'un marché de la linguistique dominé par les mondains. Des traductions, partielles il est vrai, des oeuvres de Diez ont été imprimées en Belgique où Grandgagnage apparaît comme le successeur du fondateur des études romanes. Comme Burnouf sait le travail accompli outre-Rhin, Littré correspond avec Diez dont les travaux ont été présentés, en 1841, par Jean-Jacques Ampère dans son Histoire de la formation de la langue française ${ }^{22}$. Gaston Paris rappelle, lors du banquet du centenaire de la naissance de Diez:

Il (Diez) avait en effet souffert que dans les pays romans, où ses ouvres auraient dû être accueillies avec la plus vive gratitude, elles fussent très longues à pénétrer. Ses travaux vraiment admirables de finesse et de pénétration sur la littérature provençale étaient en France comme non avenus, et l'Histoire littéraire continuait, longtemps après qu'ils avaient paru, à publier sur les troubadours des notices où l'on n'en tenait aucun compte; les traductions, d'ailleurs plus que médiocres, de deux d'entre eux par un littérateur belge restaient inconnues du public. Sa grammaire, hâtivement et maladroitement résumée, dans sa partie française, par Ampère, provoquait les sarcasmes de Génin, qui pensait écraser l'auteur sous le reproche d'avoir traité le français " comme il eût fait le sanscrit ou le persépolitain ", et ne rencontrait que le scepticisme à l'École des Chartes même, où Guessard se

21. L'E.P.H.E. fait pourtant figure de parent pauvre: pas de locaux (on leur " prête * une table de la bibliothèque de la Sorbonne), peu de crédits, des rémunérations faibles ( 1.200 à $2.000 \mathrm{~F}$ pour un professeur de l'E.P.H.E. contre $7.500 \mathrm{~F}$ pour un professeur de faculté, $5.000 \mathrm{~F}$ pour un professeur de "Langues $\mathrm{O}$ „. 2.000 à $4.000 \mathrm{~F}$ pour un professeur des Chartes).

22. L'ouvrage fait environ 400 pages (cf. G. Paris, "Grammaire hâtivement et maladroitement résumée ). 
faisait fort d'opposer à chacune des règles de Diez autant d'exemples qu'il en avait donnés pour l'appuyer ${ }^{23}$."

G. Paris impute au fonctionnement même du marché de la linguistique la marginalisation perpétuée du comparatisme, si efficiente qu'elle contraint ceux qui veulent s'initier à la grammaire historique à étudier en Allemagne, renversant le classement acquis qui situait l'enseignement supérieur français très au-dessus de son homologue allemand.

La jeunesse des professeurs de l'E.P.H.E. trahit une crise universitaire : le pouvoir est contraint de créer, contre les facultés et le marché de la linguistique traditionnel, une école dont les postes ne peuvent être pourvus par ces deux filières. En occupant précocement les quelques postes débloqués, Paris, Bréal et Brachet modifient l'ensemble des équilibres du champ, l'économie du marché de la linguistique et, pour une part, le fonctionnement social de l'enseignement supérieur. D'abord, l'absence d'attente pour une nomination à l'E.P.H.E. situe ces jeunes professeurs à égalité avec des collègues plus âgés dont l'expérience et la formation sont très différentes et les sépare des étudiants de leur génération qui reçoivent des postes en lycée ou dans des facultés de province au même âge. Cet isolement est renforcé par l'établissement de l'E.P.H.E. en marge des facultés.

Le système de reproduction de l'enseignement supérieur ne permet plus d'intégrer les acquis scientifiques: en créant une dépendance forte à l'égard des maîtres en place, il paralyse l'innovation, perpétue les dépendances, les reconnaissances et les solidarités, fige les circuits d'accès aux chaires convoitées en ralentissant les impétrants. La jeunesse des comparatistes français interdit toute reproduction fondée sur le renouvellement biologique, d'autant qu'il n'existe pas de poste d'attente et pas de filière de formation. A l'exception de M. Bréal, l'E.N.S., qui est le point de départ obligé pour toute carrière universitaire, n'a guère fourni de cadres à la première génération de romanistes et d'orientalistes scientifiques. La formation a été poursuivie dans les établissements les plus divers (École des Chartes, Collège de France, École rabbinique de Paris, Universités allemandes...) : l'orientation finale représente une rupture, une inflexion de la trajectoire de ces étudiants qui, devenus professeurs, sélectionnent leurs élèves sans contrainte de titres dès lors que leur enseignement $n$ 'est sanctionné par aucun diplôme à valeur professionnelle. Cette génération qui n'a nul maître en France doit inaugurer un rapport nouveau à ses élèves dont elle n'est vraiment séparée ni par l'âge, ni par l'apparat: faire cours à dix élèves sur un coin de table interdit le cours ex cathedra et l'autorité fondée sur les signes de l'autorité seuls. Contre le marché "civil " de la linguistique, le marché "d'état " de la linguistique se sépare du purisme, des amateurs de patois et d'argot, des critiques littéraires, des grammairiens en privilégiant l'ésotérisme scientifique. La situation se modifie brusquement avec la défaite française de 1870. Renan, dont La réforme intellectuelle et morale de la France (1871)

23. Discours reproduit dans la chronique de janvier-avril 1894 de la revue Romania. 
sert de référence à toute une génération, analyse les causes du désastre : "Dans la lutte qui vient de finir, l'infériorité de la France a été surtout intellectuelle, ce qui nous a manqué, ce n'est pas le cour, c'est la tête. L'instruction publique est un sujet d'importance radicale; l'intelligence française s'est affaiblie; il faut la fortifier [...]. Notre système d'instruction a besoin de réformes radicales [...]. C'est surtout dans l'enseignement supérieur qu'une réforme est urgente. "En même temps que le modèle fourni par l'E.P.H.E. est mis à l'honneur, tout ce qui vient d'Allemagne, fût-ce de la science, est rejeté par l'opinion, c'est-à-dire par le marché mondain de la linguistique qui se trouve conforté dans sa négation de la grammaire historique par la rancœur nationaliste. Il faudra que le pouvoir intervienne pour imposer les savants sur le marché de la linguistique : l'intervention conjointe du politique et de la science en faveur de la romanistique (et, à un moindre titre, de l'orientalisme), sur un champ paralysé par le purisme, est un symptôme : il y a désormais, en linguistique, des questions qui se posent et dont la résolution ne peut être tranchée ni par décret - ainsi que procéda la Révolution francaise -, ni par les jugements des puristes ou des académiciens.

Sous la pression des linguistes formés en Allemagne et avec l'aide des hauts fonctionnaires du ministère de l'Instruction publique, le marché de la linguistique se fracture; un ensemble financé par des crédits d'État affronte le marché international et les spécialistes de la discipline; il s'oppose à un autre ensemble, homogène du marché littéraire ou scolaire, peu à peu marginalisé jusqu'à disparaître de l'université. C'est au premier groupe des linguistes scientifiques et à son intégration dans l'université que nous allons d'abord nous intéresser.

\section{Comment devenir linguiste.}

La linguistique, de n'être pas constituée en marché objectivé, indépendant de la littérature et du purisme, semble peu attractive pour un investissement en capital symbolique de la part de quelque sujet social que ce soit dès lors qu'il peut prétendre à un droit à la parole sur la langue ${ }^{24}$. Dans l'ensemble des marchés possibles, le marché de la linguistique offre peu de profits matériels ${ }^{25}$ (grammaires scolaires, dictionnaires, manuels...) ou symboliques (Académie des Inscriptions et BellesLettres, collaboration aux journaux) : il requiert un travail important pour une reconnaissance sociale limitée, les avantages qu'on en peut retirer sont aléatoires, inconsistants. Jusqu'au dernier tiers du $\mathrm{XIX}^{\mathrm{e}}$ siècle, à l'exception des lexicographes, les concurrents en lice sur ce marché paraissent être soit des polygraphes (Girault-Duvivier s'occupe de grammaire et d'archéologie, Peignot et Lalanne de toute curiosité), soit des littérateurs (tels Nodier, Mérimée, P. Paris, Génin, F. Wey, N. de Wailly et d'autres, se consacrant à des études de langue en fonction des éditions

24. Ce qui exclut non seulement ceux qui n'ont pas eu accès aux lycées mais de nombreux provinciaux, toutes les femmes, etc.

25. Même si, ponctuellement, ils sont importants, leur nombre est limité. 
de texte ou des débats sur le français qu'il faut écrire), soit des producteurs scolaires, des auteurs de grammaire pour l'école primaire aux enseignants de l'École des Chartes. Seuls font exception les quelques orientalistes français de l'École des Langues orientales et du Collège de France qui paraissent non loin de la disparition pure et simple. Les repères manquent pour orienter le marché, constituer ses centres de représentation dans l'édition ou l'enseignement supérieur, spécifier son public, symboliser les trajectoires qui y prennent et donnent sens.

Il semble pourtant, à sommer quelques-uns des paramètres sociaux propres à la nouvelle génération de linguistes qui s'implante sur ce marché à l'orée des années 1870 , que l'on puisse reconstituer une représentation commune où se serait reconnue une forme collective de "vocation " des linguistes, c'est-à-dire une adéquation possible entre une certaine forme de capital social, culturel et éthique ${ }^{26}$ et un investissement à même d'apporter les profits spécifiques attendus par celui qui s'y engage. Et cette " vocation " ne s'inscrit plus dans les voies frayées traditionnellement : elle rejoint un univers de croyance plus large à l'intérieur duquel elle s'efforce à penser le langage. D'un mot, c'est la science qui est en cause : l'horizon de perception de l'intelligentsia, et dans tous les domaines, semble s'organiser autour de l'idée de science. L'éminente figure de Littré a pu en accroître l'effet à l'intérieur du marché de la linguistique. Les solidarités tissées avec le marché littéraire, les journaux et les salons, s'estompent : Paulin Paris ou Charles Nodier étaient liés à l'école romantique; Gaston Paris ou Michel Bréal cherchent une référence nouvelle auprès des chimistes, des jeunes historiens ou des médecins. La pensée du monde doit trouver une légitimité neuve dans la rationalité conçue selon le modèle des sciences reconnues : objectivation, clôture de l'objet, système de règles, vérification expérimentale... Les découvertes allemandes répondent à ces exigences : un groupe d'étudiants, spécialistes de grammaire historique, introduits sur le marché allemand de la linguistique, sensibilisés à la nécessité de procéder scientifiquement, se forme à la fin des années 1860. En sont exclus ceux dont la trajectoire s'est déroulée sur les marges du marché littéraire, dans la mouvance du romantisme (Charles Lénient ${ }^{27}$, Saint-René Taillandier ${ }^{28}$, Frédéric Mistral ${ }^{29}$ ), que leur retard à appréhender la nouvelle configuration du champ soit lié à leur âge - les romanistes comparatistes sont nés après 1839 ou à leur origine provinciale.

Mais l'investissement dans la science, à âge et origine géographique égaux, est un choix exclusif d'autres choix : la politique, l'administration, les carrières diplomatiques ou juridiques, le journalisme, l'armée, les affaires, la cléricature ${ }^{30}$. Aussi le choix scientifique se compose-t-il des

26. Le capital éthique serait tel qu'une partie au moins de son organisation et de son sens serait liée aux instances du moi, notamment le surmoi. Cette remarque - et l'ensemble de ce travail - doivent beaucoup à P. Encrevé.

27. Charles Lénient (1826-1908), ancien élève de l'E.N.S., suppléant de Saint-Marc Girardin à la faculté des lettres de Paris, spécialiste de littérature du Moyen Age.

28. Saint-René Taillandier (1817-1879), ancien élève de l'E.N.S., professeur aux facultés de lettres de Montpellier puis de Paris. Spécialiste de poésie provençale, membre de l'Académie française.

29. Frédéric Mistral (1830-1914): poète et lexicographe provençal.

30. Cf. T. Zeldin, Histoire des passions françaises, t. II (1978). 
traits négatifs qui ont censuré toutes les autres possibilités. A l'inverse de la cléricature, la linguistique scientifique (et la science en général) écarte toute référence à la divinité ou à la métaphysique, dans son objet, et semble dans sa pratique d'une laïcité militante puisque s'y retrouvent des catholiques (G. Paris, A. Brachet...), des protestants (Saussure, Gilliéron, Passy) et des juifs (Bréal, Arsène et James Darmesteter, Derenbourg, G. Maspero). Mais la science apparaît aussi comme une arme contre l'église; à ce titre, les jeunes idéologues républicains de la III ${ }^{e}$ République ${ }^{31}$ en font un de leurs arguments et l'on sait quelle application en fera Ferry dans son projet scolaire. De se conjoindre à la constellation politique nouvelle, la plupart des linguistes tendent à assimiler leurs valeurs à celles de la "gauche ", ce qu'induisait déjà leur "désintéressement ", leur refus d'une carrière liée à la manipulation des marchandises ou de l'argent. La remarque de Rabi ${ }^{32}$ dépasse la communauté religieuse qu'il étudie : "Après 1870 apparaît la troisième génération. Elle est libérée, épanouie, ardente, décidée, scientiste. Les pères ont été financiers, banquiers, hommes d'affaires, négociants : les fils sont intellectuels. La recherche scientifique constitue pour eux une attraction irrésistible. Ils jaillissent désormais par tribus, par castes, par familles. * Le seul trait qui semble propre à la communauté juive serait l'origine sociale, le passage du monde du commerce à celui des idées. A l'exception de Paul et Jean Passy, les autres linguistes n'appartiennent pas à des familles engagées dans les affaires.

En se situant dans la science, les romanistes s'identifient au système de valeur qui lui est alors corrélé : croyance au progrès, cosmopolitisme, athéisme (ou laïcité), toutes idées en contradiction avec celles en partage sur les autres marchés ouverts à des étudiants (armée, administration, diplomatie...). Là où dominent le conservatisme, le catholicisme et un patriotisme antijacobin, y compris dans l'Université, les linguistes sont ignorés. Mais la science les écarte aussi du journalisme et de la politique, lieux d'où l'objectivation semble bannie. Cette suite de refus a deux effets : d'une part, elle ne concerne qu'une infime minorité d'étudiants ${ }^{33}$, d'autre part, elle éparpille le recrutement entre des groupes sociaux et religieux dont la distribution antérieure du marché assurait le compartimentage. La logique de leur réunion échappe à tout modèle unitaire.

Qu'il s'agisse des romanistes ou des orientalistes, leur premier trait spécifique est la possession d'une seconde langue en fonction de laquelle ils construisent un rapport différent à la linguistique selon que cette langue est " maternelle " ou "scolaire ". Ainsi aux romanistes initiés à leur discipline en Allemagne - et dont l'avance objective qu'ils prennent sur le marché de la linguistique doit être rapportée à la germanophonie, perçue comme langue seconde par rapport au français - s'opposent les romanistes provençalistes qui stipulent dans leurs recherches le primat de l'occitan sur le français, et pas seulement à titre personnel, anecdotique. De même les orientalistes formés dans les universités d'outre-Rhin

31. Cf. C. Nicolet, L'idée républicaine en France (1982).

32. Rabi, Anatomie du judaïsme français (1962), p. 66-67.

33. Le millier d'étudiants en lettres sera atteint dans l'ensemble des facultés françaises en 1882 . 
ou profitant de leur connaissance de l'allemand pour s'imposer sur ce marché s'opposent à ceux ayant pour langue maternelle un parler d'Asie mineure, tel Joseph Halévy. A l'intérieur du champ de la linguistique en gestation, deux représentations de la langue s'affrontent selon que le linguiste s'est ou non dépris dans la science de sa constitution symbolique et imaginaire.

\section{Les romanistes}

Deux groupes sont en concurrence pour imposer sur le marché de la linguistique leur définition du champ : l'un est regroupé à Paris autour de la Revue critique, fondée en 1866, l'autre s'est formé à Montpellier en 1867-1868 comme "Société d'Étude des Langues romanes » avec la Revue des Langues romanes. Les "Parisiens ${ }^{34}$ " sont, en 1870: G. Paris (né en 1839), P. Meyer (né en 1840) et Auguste Brachet (né en 1844). Tous trois sont originaires de pays de langue d'oil - Paris est champenois, Meyer parisien et Brachet tourangeau -, de famille dont le capital symbolique prime le capital économique (professeurs ou militaires), élèves de l'enseignement supérieur (Paris et Meyer chartistes, Brachet a préparé Saint-Cyr mais y a renoncé pour des raisons de santé). Tous trois ont appris l'allemand, G. Paris parce que son père, déjà présent sur le marché de la linguistique, en sait la nécessité, A. Brachet parce qu'il appartient à une famille de militaires. Au terme d'un accord probablement implicite, G. Paris se consacre au domaine de langue d'oil et P. Meyer à l'occitan : dès 1868, la répartition transparaît dans les textes édités (Flamenca pour Meyer et La vie de Saint-Alexis pour Paris ${ }^{35}$ ).

Dans un champ caractérisé entre autres par la dispersion des trajectoires, Paris et Meyer représentent une exception : nés pratiquement la même année, ayant fait leurs études secondaires à Paris, ils se forment en même temps à l'École des Chartes et auprès de Diez et Delius à l'université de Bonn avant de fonder ensemble la Revue critique d'Histoire et de Littérature (1866) et la Romania (1872). Ils représentent un des pôles les plus stables du marché de la linguistique. A l'inverse, Brachet apparaît comme un provincial, sans formation spécifique - c'est un autodidacte en romanistique ${ }^{36}-$, contraint de substituer à la stratégie de groupe de Paris et Meyer la négociation avec la fraction la plus ouverte de l'état antérieur du marché de la linguistique : Taschereau, conservateur à la Bibliothèque nationale, qui lui confie une tâche subalterne dans ses services ${ }^{37}$, Littré qui préface sa Grammaire historique fran-

34. Le terme est excessif puisqu'il exclut des linguistes n'appartenant pas à la jeune génération (Littré, Godefroy, de Wailly) et un romaniste comme Léon Gautier (1832-1897) qui se définit à l'encontre de Paris ou Meyer dans son travail scientifique comme dans ses appréciations politiques ou religieuses (il est royaliste et catholique).

35. De même que le nom de Paris a pu interférer dans l'imaginaire du linguiste pour reconstruire une image de la langue centrée sur la capitale, de même on peut noter qu'Alexis est le prénom (le second) du père de Gaston Paris.

36. Il s'inscrit à l'École des Chartes en 1864 mais ne peut suivre assidûment les cours, étant contraint de travailler.

37. Taschereau est tourangeau et Brachet profite de son aide pour éditer: Étude sur Bruneau de Tours, trouvère du xirr siècle (1865). 
çaise ${ }^{38}$, Egger son Dictionnaire étymologique de la langue française (1870). Gaston Paris obtient sa nomination à titre de répétiteur de la conférence des langues romanes à l'E.P.H.E. en 1869 et l'associe à la traduction de la Grammaire des langues romanes de Diez dont le premier tome paraît en 1873.

L'arrivée d'Arsène Darmesteter modifie la répartition de l'équipe des romanistes parisiens. A. Darmesteter (1846-1888) est né dans une famille lorraine d'artisans : son père est venu à Paris pour que ses enfants puissent aller dans un bon lycée et à l'école rabbinique. Tout en suivant l'enseignement théologique de Zadoc Kahn, A. Darmesteter prépare un travail sur les gloses de Rashi (1040-1105): il se présente au cours de G. Paris à l'E.P.H.E. afin de s'initier à la langue médiévale et devient un auditeur attentif. Rapidement convaincu par l'étude des textes de la tradition juive que Dieu n'est pas nécessaire à l'existence du peuple juif, devenu athée, il se consacre à la lexicographie - Hatzfeld en fait le collaborateur privilégié de son Dictionnaire à partir de $1871^{39}$ - et à la romanistique. Tout en soutenant sa thèse : Traité de la formation des mots composés de la langue française $(\mathbf{1 8 7 3}){ }^{40}$, il s'intéresse aux ouvres dans lesquelles interfèrent la culture juive et les langues de la France médiévale, notamment les grammaires judéo-provençales, et entreprend une ouvre de linguiste par la critique de la Grammaire de Brachet ${ }^{41}$. Celui-ci, malade, semble mal supporter une telle remise en cause, d'autant que la qualité du travail d'A. Darmesteter le désigne comme second de G. Paris à ses dépens. Il démissionne de l'E.P.H.E. en 1872 pour devenir examinateur d'allemand à l'École polytechnique. Un riche mariage et une santé déficiente le conduisent à se retirer totalement du marché de la linguistique en 1873 (sa Grammaire française fondée sur l'histoire de la langue paraît chez Hachette ${ }^{42}$ en 1873).

A. Darmesteter remplace aussitôt Brachet à l'E.P.H.E. A la différence de Brachet dont l'activité s'orientait vers l'enseignement auquel ses grammaires étaient destinées, Darmesteter semble situer sa recherche sur le marché international de la linguistique, c'est-à-dire face aux Anglais (notamment Max Muller dont il retient la théorie darwinienne du langage) et aux Allemands (il est un de ceux qui introduisent en France les théories néo-grammairiennes développées à partir de 1876). Il est probable que l'origine juive de Darmesteter et sa formation à l'école rabbinique aient êté décisives dans sa conception du langage ${ }^{43}$ et dans sa posture scientifique. En se faisant le représentant de la France dans la concurrence internationale, il milite pour une identification de la communauté juive avec les intérêts de la France républicaine, comme ses core-

38. Parue chez Hetzel en 1867 sur recommandation de G. Paris.

39. Cf. G. Matoré, Histoire des dictionnaires français (1968).

40. Comme l'expliquera James Darmesteter, son frère, Arsène entendait démontrer que les ressources lexicales françaises étaient équivalentes à celles de l'allemand dans la composition.

41. Article paru dans la Revue critique (1874).

42. Véritable trajectoire éditoriale : l'ouvrage sur Bruneau de Tours (1866) paraît chez un petit éditeur scientifique d'origine allemande (Franck), la première grammaire chez un éditeur pour la jeunesse en 1867 (Hetzel), la seconde chez le principal éditeur scolaire en 1874 (Hachette).

43. Le travail philologique requis par les textes hébreux a pu induire une certaine heuristique linguistique. 
ligionnaires orientalistes. Il incarne aussi l'écart maximal entre la langue qu'il étudie et la langue qu'il parle puisqu'il minore continûment son dialecte au profit de l'hébreu ou de la représentation objectivante du français qu'il reconstruit : son rapport à la langue est un négatif de celui des romanistes provençalistes.

Les provençalistes ignorent l'allemand: en dépit de sympathies mutuelles - au moins jusqu'en 1872 -, leur logique les écarte de leurs concurrents parisiens. Une redéfinition scientifique de la langue ne constitue pas leur objectif en ce qu'elle ruinerait la complicité sociolinguistique au principe de leur travail. Leur attente est littéraire; au plus envisagent-ils de redéfinir un système normé d'appréciation des productions linguistiques occitanes, rétablissant un marché linguistique occitan indépendant du marché linguistique français ${ }^{44}$. Lorsqu'ils obtiennent la création de deux chaires à Montpellier en 1878, les provençalistes imposent des professeurs qui sont autodidactes en romanistique : Boucherie, professeur de lycée et spécialiste de versification, et Chabaneau, ancien receveur des postes, auteur d'une grammaire sur le dialecte nontronnais parue dans la revue savante des Charentes ${ }^{45}$. Ce seront les premiers et les derniers postes qu'obtiendront les provençalistes. Un linguiste, pourtant sensible aux problèmes de l'occitan, comme Léon Clédat ${ }^{46}$ (18501930), suit une formation auprès de Gaston Paris à l'École Pratique des Hautes Études avant d'être nommé à la faculté de Lyon en 1876. La bipolarisation de la romanistique française est accentuée par le problème du statut linguistique du provençal et la marginalisation politique et scientifique des romanistes provinciaux dont l'activité est tributaire du marché littéraire provençal.

\section{Les orientalistes}

L'orientalisme occupe une place spécifique dans le marché de la linguistique puisqu'il ne saurait dépendre d'une demande liée au marché linguistique. A ce titre, son existence paraît liée aux aléas de la politique étrangère de l'état qui assure l'intégralité de son financement jusqu'à la fin du $\mathrm{XIX}^{\mathrm{e}}$ siècle. Avec des moyens très faibles, un volant de postes restreint, un public très limité composé exclusivement de spécialistes, l'orientalisme véhicule probablement l'image la plus ancienne du savant linguiste et les figures de Sylvestre de Sacy, Langlès, Chézy, Champollion, Volney et Burnouf ont pu entretenir cette créance. A partir de 1820, un rétrécissement du marché de l'orientalisme est perceptible et seul, vers 1860, Jules Oppert (1825-1905) ${ }^{47}$ semble pouvoir affronter la concur-

44. Cf. Histoire de la littérature occitane de C. Camproux (1953).

45. En se joignant au mouvement provençaliste, le félibre Achard, dit le "Félibre des Chartes ", archiviste du Vaucluse, se consacre significativement à la littérature et non à la linguistique.

46. Clédat est originaire du Périgord et occitanophone mais il se situera du côté de la science en écrivant des grammaires et des traités de phonétique.

47. Jules Oppert a quitté l'Allemagne où il était interdit d'enseignement supérieur par une loi antisémite : émigré en France en 1847, il participe aux missions de fouilles en Mésopotamie et réussit la transcription et la traduction des inscriptions publiées par ses soins dans les 2 tomes de l'Expédition scientifique en Mésopotamie (1858-1863). 
rence internationale. Qui pouvait se sentir motivé pour investir sur un tel marché dans les années 1860-1870 étant donné son étroitesse et son déclin? D’une part des admirateurs de la littérature sanscrite tels Regnaud et Bergaigne, d'autre part des étudiants concernés, fût-ce imaginairement, par les langues et les cultures du Proche-Orient, notamment chamitosémitiques, c'est-à-dire des membres de la communauté juive.

Abel Bergaigne (1838-1888) fut répétiteur de sanscrit à l'E.P.H.E., attaché à la chaire de Bréal dont il est l'élève. Tous ses travaux portent sur la langue et la littérature sanscrites, en Inde et au Cambodge. A son objet près, sa carrière est proche de celle des universitaires : il est nommé à la Sorbonne à la chaire de * Sanscrit et grammaire comparée * en 1885, l'année de son élection à l'Académie des Inscriptions et Belles-Lettres. La trajectoire de Paul Regnaud est plus tumultueuse puisqu'il commence par être employé de forge et marchand de fer avant de se consacrer au sanscrit : à quarante et un ans, en 1879 , il reçoit la chaire de sanscrit de l'université de Lyon. Son œuvre, entreprise sur la littérature védique, s'étend à la philosophie du langage dans une optique darwinienne ${ }^{48}$ où se retrouve l'influence de Max Muller ${ }^{49}$.

Il nous est encore difficile de comprendre les raisons qui conduisirent Regnaud ou Bergaigne vers l'orientalisme. Tel n'est pas le cas pour Hartwig Derenbourg, James Darmesteter, Gaston Maspero et Joseph Halévy, voire Michel Bréal. Bréal (1832-1915) est né en Allemagne rhénane dans une famille juive française; élève à l'École normale supérieure, il étudie la grammaire comparée à Berlin et obtient en 1864 la conférence de "Langues et littératures de l'Inde " qu'il transforme en conférence de grammaire comparée. Appelé à l'E.P.H.E. dès sa fondation, il est le maître de Bergaigne, de James Darmesteter, d'Antoine Meillet. Fondateur de la "Société de linguistique de Paris " en 1865, il collabore étroitement avec les fonctionnaires du ministère de l'Instruction publique tout en poursuivant une cuvre de linguiste - il jette les bases de la sémantique dans un cycle de conférences au Collège de France en 1868 -, de comparatiste et d'orientaliste dans laquelle il semble soucieux de penser la langue indépendamment de la littérature et sans respecter le cloisonnement du marché de la linguistique qui sépare différents domaines dans l'ensemble indo-européen. M. Bréal représente la structure idéale de capital symbolique puisqu'il cumule les traits les plus traditionnels de la connaissance scolaire - étude du latin et du grec, scolarité en lycée parisien et à l'E.N.S. - et les acquis les plus marginaux - connaissance de l'allemand, études à Berlin - mais les plus efficaces dans l'état de vacuité du marché de l'orientalisme. Bréal est appelé au Collège de France à vingt-deux ans; il est responsable du secteur linguistique de l'E.P.H.E. à vingt-six ans! L'empan de Bréal sur le marché de la linguistique semble lié à un investissement en capital qui anticipait sur une demande que la connaissance du marché allemand de la linguistique rendait prévisible en France, dans ce domaine.

48. Cf. Essais de linguistique évolutionniste (1886).

49. Cf. "Rig Veda" et les origines de la mythologie indo-européenne (1892). 
Oppert, Derenbourg, Darmesteter, Maspero et Halévy ne bénéficient pas d'opportunités aussi rapidement gratifiantes. Venu tôt en France, Oppert a pu s'imposer en réussissant la traduction des textes assyriens : promu au Collège de France en 1874, il met en évidence à partir de 1880 lorigine sumérienne de la transcription cunéiforme. Joseph Halévy appartient à une communauté d'Andrinople : émigré en France, il travaille sur l'assyrien et le babylonien avant de collecter des inscriptions en alphabet sémitique, en moab, se déguisant pour ce faire en juif pauvre et traversant l'Arabie. Il conscitua, au péril de sa vie, un corpus de sept cents numéros et continua son œuvre par l'étude du berbère ${ }^{\text {so }}$.

Hartwig Derenbourg est né en 1844, James Darmesteter et Gaston Maspero en 1846. Tous trois ont reçu une formation secondaire à Paris et appartiennent à des familles juives intégrées, soucieuses de conserver une appartenance religieuse et culturelle et d'assimiler, à titre de stratégie collective d'une fraction du judaïsme français, l'humanisme rationaliste et universaliste de la Révolution française comme continuation d'un positivisme juif laïque et instrument de libération et de promotion des communautés de la diaspora. Cette orientation prolonge l'œuvre d'Adolphe Crémieux (1796-1880), né dans une famille juive jacobine du Midi, membre puis président (de 1843 à 1845) du Consistoire central, fondateur en 1860 de l'Alliance israélite universelle qui se propose, dans le cadre du missionnarisme français, d'assurer l'ouverture au monde moderne des juifs du Proche-Orient grâce à la culture française. L'A.I.U. ouvre des centres en Égypte (1862), Palestine (1863), au Maghreb, au Liban, en Syrie ${ }^{51}$... Dans le champ de l'orientalisme, le choix de Maspero vers l'égyptologie ou celui de Derenbourg vers l'arabe, à un moindre titre les études iraniennes de J. Darmesteter, réalisent une aspiration dont l'enjeu est inscrit d'abord dans le capital éthique, c'est-à-dire dans l'habitus, le mandat reçu par ces jeunes intellectuels dans une partie de la bourgeoisie juive en France au XIX ${ }^{e}$ siècle. Hartwig Derenbourg est le fils de Joseph Derenbourg, un orientaliste originaire de Mayence, professeur dans un grand lycée parisien. Il suit les cours de l'arabisant Reinaud à Paris et complète sa formation à Göttingen auprès d'Ewald. D'abord attaché à la Bibliothèque nationale, il enseigne l'arabe à Langues $O$. à partir de 1879 et à l'E.P.H.E. Il se consacre à la partie linguistique du champ en publiant notamment son Essai sur les formes des pluriels arabes (1867). Gaston Maspero (1846-1916) est né à Paris; élève de l'E.N.S., il envisage d'abord d'étudier les civilisations précolombiennes mais opte finalement pour la culture égyptienne et obtient, en 1869, la charge de répétiteur d'archéologie et de philologie égyptiennes à l'E.P.H.E. Il travaille en collaboration avec les linguistes, y compris les romanistes, et donne Des formes de la conjugaison en égyptien ancien, en démotique et en copte (1871). Dès 1874, E. de Rougé lui demande de le suppléer dans sa chaire du Collège de

50. Sur Halévy, cf. The discovery of language - linguistic science in the nineteenth century d'H. Pedersen (première édition en danois : 1932).

51. C'est aussi Crémieux qui fera adopter la naturalisation des juifs algériens en 1870 . Ses efforts se comprennent mieux si l'on se rappelle que la France est un des rares pays à avoir aboli toute discrimination, avec l'Angleterre (il se fonde en 1871 une * Anglo-Jewish Association * pour concurrencer l'A.I.U.). Cf. Rabi, Anatomie du judaïsme français (1962). 
France avant qu'il ne soit envoyé è Égypte en remplacement de Mariette pour diriger les fouilles, en $1880^{52}$.

James Darmesteter (1849-1894) suit une carrière parallèle à celle de son frère : il abandonne les études rabbiniques et devient le collaborateur de Michel Bréal en se spécialisant dans la culture iranienne, mais il dévie sa recherche des questions linguistiques aux problèmes de mythologie comparée. Il participe, avec Arsène et Zadoc Kahn, à la création de la Revue des Études juives comme organe de recherche scientifique (et laïque) de la communauté. La présence de nombreux juifs sur le marché de la linguistique semble avoir eu plusieurs effets : approfondir la séparation avec le marché linguistique en déterritorialisant l'étude de la langue, renforcer l'importation des connaissances allemandes, rompre la tradition catholique et réactionnaire des universités, imposer une approche neuve de la question de la langue. La nouveauté du champ permit aux juifs d'y pénétrer en profitant de la caducité des stratégies de reproduction fondées sur un ou plusieurs critères de la logique sociale - appartenance à un même groupe familial, social, géographique, politique, religieux... - au profit des critères fondés sur le capital scientifique, sur un capital objectivable en ce qu'il est lié à une personne par un apprentissage volontaire et non par une désignation du corps social. Par l'attitude collective qu'elle adoptait à l'égard de sa double identité, religieuse et nationale, la communauté juive en France semble disposer d'une avance homologique, au moins pour la fraction qui tentait de retranscrire son identité en termes sociologiques - substituant la notion de culture à celle de race -, contre les théories biologiques ou métaphysiques ${ }^{53}$. Tout se passe comme si, en effet, Maspero, Derenbourg ou les Darmesteter reproduisaient dans leur travail de construction scientifique du marché de la linguistique, et selon les contraintes propres du marché ${ }^{54}$, la démarche déjà accomplie par leur famille pour repenser le judaïsme hors de la sphère religieuse afin de le retranscrire dans l'idéologie républicaine inspirée des principes de 1789.

Ces linguistes sont amenés à concevoir leur situation (et leur mission) comme liée aux deux communautés qu'ils reconnaissent être leurs : rédimer les juifs orientaux, comme l'a fait juridiquement Crémieux, par la culture française au nom de l'unité de la diaspora et offrir à la France l'ouvre de tous les juifs initiés à sa culture. Ainsi concevrait-on mieux l'ambition avouée d'A. Darmesteter d'être le premier juif élu à l'Académie française, afin de représenter ses coreligionnaires auprès de la plus " française " des institutions et de représenter la France auprès des juifs ${ }^{55}$. Ce

52. C'est Maspero qui dégage le Sphinx et le temple de Louqsor.

53. Il faudrait, pour rendre compte de ce mouvement d'idées, rappeler le rôle de Durkheim dans la fondation de la sociologie francaise et l'opposer au travail de Rolland, Sébillot ou Gaidoz, les premiers folkloristes français, ou à celui de Broca pour l'anthropologie.

54. Contraintes qui vont à leur tour favoriser cette même fraction de la communauté juive dans la mesure où elle parle allemand, elle vit à Paris (ce qui exclut les juifs de la dialectologie), elle mène des études supérieures...

55. Il reste à montrer comment un tel projet fondé sur la langue se noue à la langue, comment telle erreur d'A. Darmesteter sur l'histoire du mot * daintiers * n'est rien de plus qu'un lapsus scientifique où se trahit l'imaginaire. 
projet sera considérablement ébranlé par la montée de l'antisémitisme, de La France juive de Drumont (1886) à l'affaire Dreyfus.

\section{Les linguistes et leur marché}

La bipolarisation du marché de la linguistique entre romanistique et orientalisme ${ }^{56}$ détermine un espace à l'intérieur duquel il est possible de saisir une stratégie collective de contrôle du marché de la linguistique et son organisation à travers un ensemble de sociétés et de revues. Une des tendances dominantes de ce dispositif serait son progressif transfert d'une prise en charge "civile" (bénévolat des charges, appel au mécénat, absence de locaux spécifiques) à une prise en charge "étatique " (financement de l'édition de périodiques et d'ouvrages, subventions, prêt de bureaux), assurée généralement à travers certaines institutions spécialisées. Une telle politique avait déjà conduit à l'édition de la " Bibliothèque de l'École des Chartes - recueil d'érudition publié par la société de l'École des Chartes et consacré spécialement à l'étude du Moyen Age " (1839) sur fonds publics. D'où l'action de certains linguistes (Bréal, Meyer, Paris...) afin d'occuper certains postes particuliers leur permettant de transformer le marché de la linguistique. Alors que la Société pour l'Étude des Langues romanes demeure, dans son recrutement et dans son fonctionnement, indépendante des ressources de l'Instruction publique, les directeurs de Romania et des Mémoires de la Société de linguistique de Paris sont des fonctionnaires. Le soutien apporté par le pouvoir républicain à certains groupes, à certaines sociétés pourrait signifier que la redéfinition scientifique du marché de la linguistique répondait, au moins en partie, à des attentes du marché politique construites par les hauts fonctionnaires du ministère de l'Instruction publique ${ }^{57}$.

La "Société de l'Enseignement supérieur " paraît particulièrement représentative de l'interaction entre la fraction la plus avancée des linguistes dans la transformation de leur champ et le groupe sociopolitique inséré dans l'administration universitaire afin de promouvoir une politique nouvelle de l'éducation.

\section{La Société de l'Enseignement supérieur}

Fondée en 1878 par vingt-quatre scientifiques de renom, dont Michel Bréal et Gaston Paris ${ }^{58}$, la S.E.S. constitue un groupe de pression aggior-

56. Cette bipolarisation existait avant 1870 mais elle fracturait le marché de la linguistique au lieu d'y dessiner une plage scientifique unique.

57. Ces hauts fonctionnaires ont des caractéristiques sociales proches de celles des linguistes et leur situation au ministère assure une continuité de leur ceuvre supérieure à celle des ministres.

58. Les autres littéraires sont Ernest Renan, le latiniste Gaston Boissier, les historiens Fustel de Coulanges et G. Monod. 
namentiste représentatif d'une nouvelle génération d'universitaires. La S.E.S. cherche à imposer une redéfinition du système universitaire en privilégiant la définition scientifique contre les positions traditionnelles d'autorité détenues par des professeurs âgés, rescapés de purges politiques successives et dispensés, par la cooptation, des jugements et des sanctions du marché de la production des biens symboliques. Comme le note George Weisz: "La plupart des membres fondateurs avaient atteint leur statut intellectuel en poursuivant des carrières consacrées à la recherche, secteur marginal dans le système traditionnel des études ${ }^{59}$.

L'identité de vue est totale entre romanistes, orientalistes et hommes politiques de la III République : patriotisme, création d'une grande université française, lutte contre l'Eglise et les conservateurs majoritaires dans les facultés, contrôle du primaire et du secondaire par le supérieur, triomphe de la science et de la science française...

L’adhésion des comparatistes à la S.E.S. correspond à leur stratégie de ruptures sociale et scientifique : ils semblent suffisamment implantés en 1878 pour obtenir d'être reconnus des autres secteurs de la recherche, demander la redéfinition à leur avantage du dispositif universitaire (contenus, carrières, recrutements) et apparaître comme les interlocuteurs privilégiés du pouvoir qui leur apporte sa caution, comme si dix ans après la fondation de l'E.P.H.E. la situation avait suffisamment évolué pour transposer le modèle de Duruy à l'échelle du pays. En dix ans, le petit noyau des romanistes et orientalistes parisiens a pu acquérir le monopole de la représentation du marché scientifique de la linguistique à travers revues et sociétés.

\section{Revues et sociétés}

" La Revue critique a porté le dernier coup à l'école des rhéteurs, pâles imitateurs de Villemain $(\dagger 1870)$. Ses premiers rédacteurs, Paris, Meyer, Bréal, Morel, Thurot, Weil, Reuss, donnant et faisant des exemples, ont bien mérité de l'érudition française. Aucune revue n'a rendu plus d'arrêts ni rendu plus de services ${ }^{60}$. "Cette appréciation de S. Reinach rappelle le rôle de la revue dans l'action des comparatistes avant toute implantation dans l'université ${ }^{61}$. Meyer prétendit même que Duruy avait recruté les enseignants de l'E.P.H.E. exclusivement parmi les rédacteurs de la revue, à la fois historique, littéraire et linguistique. Probablement pressés par la concurrence de la Revue des Langues romanes, les romanistes parisiens, tout en restant présents dans la Revue critique, fondent Romania en 1872, profitant d'une forte demande sociale concernant les civilisations romanes (opposées aux civilisations germaniques). Ils déter-

59. "La réforme de l'Enseignement supérieur sous la IIle République 1878-1896 ^, dans Alain Drouard et al., Processus de changements et mouvements de réforme dans l'Enseignement supérieur français (1978).

60. Salomon Reinach, Manuel de philologie classique (1879), p. 13 (note). S. Reinach (1858-1932), archéologue et philologue classique, est le frère du publiciste, Joseph, proche de Gambetta.

61. La Revue critique est de plus éditée par un libraire, récemment émigré d'Allemagne et sans moyens financiers: Franck. 
minent trois domaines relevant de leurs compétences : la linguistique romane, la littérature médiévale et les études ethnographiques régionales, traçant un partage du marché qui réserve aux orientalistes la linguistique indo-européenne et chamito-sémitique (et par extension la linguistique générale), la littérature et la mythologie orientales ${ }^{62}$ et l'ethnologie.

\section{A. Les patois}

Les romanistes semblent chercher à s'approprier toute la partie du marché de la linguistique consacrée aux patois, nébuleuse de dilettantes dispersés dans toute la France dont la production, confidentielle, paraît dans les revues savantes locales ou sous forme de brochures à tirage restreint. Réunir ces dialectologues de terrain était un des projets de Burgaud des Marets dans les années 1860 mais la maladie l'avait empêché d'y parvenir. Romania et la Revue des Langues romanes en relancent l'idée mais les comparatistes essuient un échec avec leur "Société des parlers de France " dans la mesure où il leur est difficile d'entrer en rapport avec des érudits provinciaux, de leur imposer des directives de travail scientifique et de briser l'intrication sentimentale ou du moins subjective de leur investissement dans le travail dialectologique ${ }^{63}$. En dépit de tentatives répétées (appel dans la Romania, association avec la société savante de Nancy, création d'un prix, adresse aux instituteurs se succèdent de 1872 à 1888), la disparité entre marché scientifique de la linguistique et marché "amateur " interdit toute cuvre commune. La dialectologie sera constituée en rupture du marché " amateur " à partir de 1883 (premiers cours de Gilliéron à l'E.P.H.E. ${ }^{64}$ ).

Sur le marché linguistique, les dialectes représentent la strate la plus négativement marquée en ce qu'ils cumulent les traits disqualifiants de leurs locuteurs - à la fois plus âgés, plus provinciaux, plus campagnards, moins scolarisés, moins "masculins "6.5 que les locuteurs du français - et une ressemblance avec la langue française au principe des jugements portés sur les " patois". La différence, perçue comme corruption, requiert, pour être "corrigée ", la généralisation de l'instruction publique. Si les romanistes tentent de procéder à une réévaluation des patois comme témoignage de l'évolution et du morcellement de la Romania, ils refusent de leur accorder une existence autonome dans la situation linguistique de la France moderne.

La logique scientifique du marché de la linguistique (étayée sur la reconnaissance génétique des langues), constituant les patois en objet d'analyse, réfutait la logique sociale en œuvre sur le marché linguistique. Cependant, aucun dialectologue ne semble avoir occupé de position centrale dans le champ de la linguistique, probablement en raison d'une

62. Oriental désignant les langues indo-iraniennes et sémitiques seules.

63. Les principaux dialectologues non professionnels sont tous des nobles (Chambure, Jaubert, Montesson) ou des royalistes catholiques, cause supplémentaire d'antinomie.

64. Deux dilettantes accéderont pourtant au marché professionnel : Clair Tisseur et Edmond Edmont, l'enquêteur de Gilliéron pour l'A.L.F.

65. Les hommes étant plus souvent scolarisés que les femmes sont plus souvent francophones. 
caractéristique sociale constituée négativement sur ce champ ${ }^{66}$. Jules Gilliéron est étranger (Suisse), Jean-Pierre Rousselot appartient au clergé séculier ${ }^{67}$, Léon Clédat poursuit toute sa carrière à Lyon, loin du marché parisien. De tels handicaps, à situer dans la poursuite de la lutte contre les patois à travers la mise en place de l'école primaire, peuvent expliquer l'échec de toutes les revues consacrées aux patois:

- Revue des patois de Clédat (1887-1888): l'échec de ce périodique contraint Clédat à s'associer avec Gilliéron et à redéfinir son objet en le remplaçant par la Revue de philologie française et provençale (18891896) puis par la Revue de philologie française et de littérature (1887 sq.) ${ }^{68}$.

- Revue des patois gallo-romans de Gilliéron et Rousselot (1887-1893), dernière tentative pour fédérer les producteurs non professionnels du marché de la linguistique ${ }^{69}$.

- Bulletin de la Société des parlers de France (1893-1899) ${ }^{70}$.

- Bulletin des parlers normands de Charles Guerlin de Guer (1897-1903) dont le siège est à Caen.

Les revues belges (Bulletin du Dictionnaire général de la langue wallonne, Revue de Dialectologie romane, Bulletin de l'Académie royale de langue et de littérature françaises), suisse (Bulletin du Glossaire des patois de la Suisse romande) ou canadienne (Bulletin du parler français au Canada), quoique plus récentes (elles sont créées entre 1900 et 1910 pour la plupart), ne parviennent pas non plus à s'imposer, probablement asphyxiées par l'étroitesse du marché.

\section{B. Études celtiques et folklore}

Devant les difficultés rencontrées dans l'organisation des études dialectologiques, les comparatistes s'ouvriront à la recherche entreprise dans des champs connexes, les études celtiques (la Revue celtique est fondée en 1876 par d'anciens chartistes tel D'Arbois de Jubainville ${ }^{71}$ ou des élèves de l'E.P.H.E. comme Gaidoz) ou la folkloristique. Il serait temps qu'une étude d'ensemble restitue l'apparition en France des recherches de folklore, une vingtaine d'années après que l'Italie, l'Angleterre et l'Allemagne ont commencé, abandonnant les bibliothèques où Ludovic Lalanne et Francisque Michel conduisaient leur travail, pour entreprendre un travail d'enquête sur le terrain (Paul Sébillot, Eugène Rolland,

66. Qui surdétermine une marginalisation consécutive à l'extraction provinciale des dialectologues qui essaient de reconvertir la part la plus dévalorisée de leur capital symbolique en élément de leur capital scientifique.

67. Ce qui détermine non seulement une profession mais un profil de carrière (du séminaire à l'Institut catholique de Paris) et une relative autonomie à l'égard de l'université d'État grâce aux subsides ecclésiastiques (il entre au Collège de France en 1897 comme préparateur du laboratoire de phonétique).

68. Située à Paris, cette revue poursuit sa numérotation sous ses titres successifs (la Revue des patois constitue les tomes I et II, la Revue de philologie française et provençale les tomes III à X, etc.) dont l'intitulé révèle la réorientation imposée par le champ : des patois à la littérature avec une transition par la philologie.

69. Située à Paris, elle propose un système de transcription phonétique des patois toujours utilisé par les romanistes.

70. Située à Paris, elle bénéficie du soutien de G. Paris, P. Meyer, J. Gilliēron...

71. D'Arbois de Jubainville Marie-Henri (1827-1910), chartiste, archiviste de l'Aube, professeur de langue et littérature celtiques au Collège de France (1882). 
Henri Gaidoz ${ }^{72}$ ). Après quelques échecs - Mélusine (1877), " revue de mythologie, littérature populaire, traditions et usages "-, les folkloristes se regroupent autour de la Revue des Traditions populaires (1866 sq.) mais acquièrent une autonomie qui semble les séparer des linguistes.

Il semblerait que le capital scientifique dont disposent les comparatistes ne suffise pas à structurer le marché " amateur " de la linguistique dans la mesure où le pouvoir ne saurait y trouver prise dès lors qu'il participe objectivement à sa dévalorisation en n'accordant aucune reconnaissance aux patois dans les situations officielles (école, armée, administration...) et se prive de toute possibilité de hiérarchisation en n'offrant aucun poste auquel serait dévolue l'autorité sur ces idiomes.

\section{Littérature médiévale}

C'est en juillet 1874 que $P$. Meyer et G. Paris annoncent la création prochaine d'une société pour la publication de textes en ancien français. Des réunions préparatoires à l'Assemblée générale de fondation du 15 avril 1875 permettent aux initiateurs de la S.A.T.F. ("Société des Anciens Textes Français ${ }^{73}$ ") d'assurer à leur société l'assentiment de tous les romanistes, la collaboration des archivistes et bibliothécaires et des sources de financement. En portant à la présidence Paulin Paris, les comparatistes ne rendent pas seulement hommage à son fils, Gaston, ils suspendent leurs rivalités avec les maîtres de la génération précédente à laquelle ils concèdent une vice-présidence, celle d'E. Egger ${ }^{74}$, et des titres de " conseillers " (Didot, Léon Gautier, J. de Laborde, Marty-Laveaux...). Afin de bénéficier de l'aide des bibliothécaires qui contrôlent les fonds manuscrits et toutes les archives, les professeurs de l'École des Chartes - dont Paul Meyer, secrétaire de la S.A.T.F. -, les conservateurs de la Bibliothèque nationale - tel Michelant ${ }^{75}$-, sont intégrés dans le conseil. Enfin le mécénat est représenté par deux amateurs, propriétaires de collections de manuscrits et d'incunables, le marquis de Queux de Saint-Hilaire, nommé administrateur et le baron James de Rothschild, nommé trésorier. La S.A.T.F. rencontre un réel succès : l'année de sa fondation, elle compte déjà trois cent trente membres et publie un petit bulletin trimestriel ${ }^{76}$. Elle attribue un monopole de l'édition de textes à des romanistes français ${ }^{77}$ et leur assure une impression de qualité, un accès facilité aux fonds des bibliothèques publiques ou privées, une certaine notoriété en raison de la rigueur des choix. Si deux générations antagonistes de médiévistes sont ensemble dans la S.A.T.F., seuls les comparatistes se chargent des éditions. En regard de l'échec de la "Société des parlers de

72. Gaidoz Henri (1842-1932), élève à l'E.P.H.E., il en sera le directeur de langue et de littérature celtiques. Spécialiste de civilisation gauloise et de folklore breton.

73. Et non "Société des textes en ancien français : ainsi les textes en occitan du Moyen Age relèvent de la S.A.T.F.

74. L'autre vice-présidence est confiée à Gaston Paris.

75. Natalis de Wailly, dont le rôle fut considérable dans la fondation de la société, refusa toute charge dans le bureau ou le conseil.

76. Volontairement réduit pour ne pas concurrencer Romania.

77. D'où des difficultés avec les romanistes allemands (dès 1875 avec Wendelin Foerster pour une édition d'Aïol parue en Allemagne avant le travail de Normand et Raynaud pour la S.A.T.F. par exemple. Cf. Chroniques de Romania, 1876, sq passim). 
France ", la réussite de la S.A.T.F. semble corrélée à une prise meilleure des romanistes et universitaires parisiens sur un marché où l'éclatement, l'atomisation est moindre, qu'il s'agisse des savants, presque tous concentrés à Paris, des sources documentaires ou de la nature même de l'objet; l'approche des manuscrits se prête mieux à l'imposition d'un monopole que la connaissance des patois et le travail sur des écritures paraît moins labile que les indécisions de l'oral. Bref, la S.A.T.F. est le domaine où les résultats, sur le marché de la linguistique, semblent les plus facilement reconnus et trouvent une unanimité que l'on ne retrouve pas dans d'autres secteurs; et elle se donne pour tâche de rendre à la France sa littérature. L'échec le plus net est dans le domaine des patois comme si la science reproduisait, dans l'inégalité de ses fortunes, les jugements acquis, à l'extérieur du marché de la linguistique, sur le marché linguistique.

\section{La Société de linguistique de Paris}

Les orientalistes, tenus à l'écart des Sociétés romanistes, se retrouvent principalement à la "Société de Linguistique de Paris " dont la fondation, vers 1865 , avait réuni deux générations de linguistes dont les cuvres divergent selon qu'ils refusent, pour les plus anciens, ou acceptent, pour les plus jeunes, les enseignements de la grammaire comparée. Vers 1870 , les comparatistes dominent la société et les publications, Bulletin et Mémoires, reflètent l'autorité qu'y ont acquis les orientalistes formés à l'école allemande, notamment Michel Bréal. Même si de nombreux romanistes adhèrent à la "Société de Linguistique de Paris", celle-ci semble détachée du marché linguistique et même des jugements qui s'y portent, de la littérature nationale et de l'horizon des interrogations sociales dont la romanistique fait son univers. La S.L.P., dont les administrateurs sont liés aux centres de recherche les plus actifs (E.P.H.E., Collège de France), apparaît comme le représentant scientifique de la France - la société est reconnue d'utilité publique dans les années 70 - sur le marché international de la linguistique.

L'agencement des revues et sociétés du marché de la linguistique tend à constituer une hiérarchie, indépendante des postes du marché universitaire, dans l'attente d'une reconnaissance assurant l'accès des comparatistes aux postes de l'enseignement supérieur. Pour appréhender l'ampleur des investissements réalisés par les linguistes, il reste à mentionner leur élection à l'Académie des Inscriptions et Belles-Lettres, au "Comité d'Histoire de la Littérature française " de l'Académie française et leur rôle dans l'édition, à la "Bibliothèque de l'École des Chartes * et à la "Bibliothèque de l'École pratique des Hautes Études ". L'efficacité de ce dispositif semble devoir pourtant tenir plus des attentes constituées en regard dans le champ intellectuel ou politique que de son extrême rationalisation ou de sa seule avance scientifique, tant la question de la langue - ou des langues - ne saurait être épuisée par le marché de la linguistique. 


\section{Le langage comme enjeu politique}

\section{Langue et nation}

Alors que l'idée de " nation " semblait s'adapter à la France et n'y plus faire problème, la guerre de 70, et notamment la perte de l'AlsaceLorraine, interrogent l'intelligentsia sur les principes et la consistance de l'unité de la France. Dès la débâcle, un observateur patenté du marché intellectuel comme Edmond de Goncourt s'interroge :

* D’un autre côté, la prétention de Belleville de vouloir despotiser la France ne pourrait-elle amener une résurrection des anciennes provinces, déjà blessées de la centralisation des règnes derniers, amener cette chose: un démembrement de la France, dont le germe existe déjà ce matin dans l'affiche de la Bretagne ${ }^{78}$ ? Et six jours plus tard : " En montant, la conversation de Nefftzer va à la dislocation de la France [...]: "Moi, je suis Germain, complètement Germain, dit Nefftzer, je défends la France seulement par devoir. Mais je ne m'abuse pas, le jour n'est peut-être pas loin où vous reverrez une république phocéenne, un grand duché d'Aquitaine, un grand-duché de Bretagne ${ }^{79}$." "

La renaissance provençale ou celtique paraît prémonitoire d'un morcellement qui serait fondé - et les exemples que choisit Nefftzer en témoignent - sur la langue, breton ou occitan. Le débat entre romanistes parisiens et provençaux sur le statut de l'occitan puise sa virulence dans l'enjeu politique qu'il symbolise. En rendant à son parler une écriture et une littérature, le Félibrige peut construire un principe d'identification en rupture avec celui du " français ". Même si Mistral, le plus emblématique des félibres, a imposé un dialecte particulièrement excentré dans une graphie réductrice ${ }^{80}$, le mouvement occitan bénéficie d'une reconnaissance non seulement sur le marché littéraire mais sur le marché scientifique - grâce à la Société pour l'Étude des Langues romanes ou à des personnalités comme Jean-Henri Fabre - ou politique - groupes républicains fédéralistes de Marseille, contacts avec les représentants de la renaissance catalane.

Paul Meyer, et les romanistes parisiens refusent de considérer le provençal comme une langue. Centrant la langue française sur Paris où la vie de cour et la littérature ont épuré et fixé la langue, ils conçoivent les idiomes romans comme la transformation progressive par d'infimes variations du dialecte de l'Ile-de-France. Comme celui-ci n'est pas attesté dans les débuts littéraires de la langue vulgaire, les romanistes doivent

78. Edmond de Goncourt, Journal, jeudi 3 novembre 1870.

79. Ibid., mercredi 9 novembre 1870 .

80. Mistral a utilisé, sur les recommandations pressantes de Roumanille, les lettres-sons du français pour la transcription de son dialecte de Maillane, rendant plus difficile la constitution d'un occitan littéraire commun à l'ensemble des pays d'oc. Mieux disposé à l'égard de la philologie, comme le montre son ouvre lexicographique, Mistral aurait souhaité une graphie indépendante de l'orthographe française. 
le reconstruire en prenant pour la première fois leur autonomie face aux textes, en s'appuyant sur la dialectologie et la phonétique. Idiome artificiellement restitué, le francien est présenté comme la forme propre au français du $\mathrm{VII}^{e}$ au XII ${ }^{e}$ siècle et comme le centre de référence des variations dialectales, de plus en plus marquées au fur et à mesure que la distance s'accroît de ce pôle aux marches du français sans que jamais rupture ne se dessine. L'étude des parlers d'oïl, par le comte Jaubert par exemple, leur présence dans la littérature, avec George Sand, restituent une continuité dont prennent argument les romanistes parisiens qui, atomisant les dialectes, leur interdisent toute prétention à concurrencer la langue française.

En centrant le débat, fût-ce nominalement, sur le " provençal ", les linguistes s'accordaient sur la démonstration à produire pour trancher le débat : qu'il existait une ligne de séparation, matérialisable sur une carte, où l'intercompréhension ne jouait plus entre les parties séparées à l'endroit même de la séparation. Ils en rendaient la démonstration presque impossible dans la mesure où cette ligne est à peu près repérable de Bordeaux à Roanne mais diluée par la dispersion des " isophones * de Roanne à la frontière italienne ${ }^{81}$. Un élève de Diez, le plus grand linguiste italien de son temps, G.-I. Ascoli, s'intéresse à ce débat. Partisan déclaré de la phonétique, il est considéré par Diez comme son meilleur disciple et entend porter un jugement sur toutes les questions de romanistique. L'analyse qu'il fait de la situation linguistique de la France reflète les conditions particulières de l'unité italienne obtenue comme reconnaissance d'une unité, entre autres, linguistique mais respectueuse des différences dialectales, jusque dans la littérature. Dans sa revue, Archivio Glottologico Italiano ${ }^{82}$, Ascoli prend parti pour la thèse des deux langues, française et occitane, en marquant l'unité du provençal au-delà des particularités de ses dialectes. Il appuie sa théorie d'une démonstration phonétique qui revient à attribuer une fixation de l'occitan deux à trois siècles avant le français. De 1874 à 1877, Meyer répond dans Romania aux arguments de son ancien condisciple de Bonn en s'appuyant sur l'échec de la mission Bringuier-Tourtoulon ${ }^{83}$. Au terme de cette polémique, deux concepts sont apparus : l'un, forgé par Ascoli, celui de " francoprovençal "; l'autre, défendu par Paul Meyer et Gaston Paris, le parler "gallo-roman". Ascoli reconnaît la séparation des isophones dans le Centre-Est de la France mais il isole deux faisceaux plus marqués au nord et au sud de Lyon et, entre deux, il retrouve suffisamment de traits propres à la langue qui s'y parle pour la caractériser d'un nom, en incluant dans sa sphère d'influence une partie de la Suisse romande et dans son histoire des documents écrits du Lyonnais médiéval. Cette

81. En fait, jusqu'à Roanne, deux zones sont loin d'être nettement séparées (Charente et Creuse) cependant qu'il existe des isolats d'oïl en pays d'oc et réciproquement.

82. Fondée au même moment que Romania qui annonce sa création en spécifiant : "pour l'ètude des dialectes italiens $\%$. Créée à Milan.

83. Octave Bringuier est un poète proche du Félibrige, le vicomte Charles de Tourtoulon un érudit du Midi. Partisans de la langue d'oc, ils sont investis en 1873 d'une " mission * officielle chargée de délimiter la frontière des langues et commencent leur travail à Bordeaux. Ils l'arrêtent l'année suivante au sud de Nevers, incapables de poursuivre le tracé de la démarcation. 
division linguistique n'est guère défendue par les provençalistes qui perçoivent le "franco-provençal " comme un dialecte de langue d'oïl; elle est récusée par l'équipe de Romania.

L'unité de langue ou de dialecte étant rendue impossible par la pulvérisation des faits linguistiques, si ce n'est en un temps révolu ${ }^{84}$, une organisation privilégiant le français littéraire comme terme de référence est esquissée à partir du concept de "gallo-roman ", décalqué sur "gallo-romain ", et censé sous-tendre la communauté des parlers romans usités en France. En retournant à un état antérieur aux invasions germaniques et en soudant en un mot les deux composantes non allemandes de l'histoire de France, les romanistes entendent en même temps se situer face à leurs concurrents d'outre-Rhin et parmi les écoles romanes. Un des rares traits qui pourra être considéré comme gallo-roman est la palatalisation du $/ \mathrm{u} /{ }^{85}$. Cette référence au " substrat " gaulois est facilitée par les travaux de l'école historique qui tend à privilégier cette part de ['héritage dans le patrimoine reconstitué : Henri Martin et Camille Jullian en sont les deux principaux représentants. C'est aussi une des raisons d'intervention de la Revue celtique dont les animateurs, Gaidoz, Luzel, Loth, Dottin et d'Arbois de Jubainville, s'attachent à retrouver, notamment dans la culture bretonne, les traits propres à une civilisation fondatrice de la spécificité française ${ }^{86}$. Dans sa constitution, le marché de la linguistique avait à répondre à la question de la nation en même temps et au même titre qu'il devait créer en France un centre scientifique d'étude de la grammaire comparée.

\section{Langue, race, terre}

Paradoxalement, les romanistes parisiens, ardents défenseurs de la grammaire comparée, d'une science élaborée en Allemagne, sont amenés à s'opposer aux linguistes allemands qui présupposent l'identité de la langue et de la nation, du "Volk ", notion raciale.

Si Louvois eût vécu de nos jours, il eût allégué quelque principe moderne : il eût prétendu que la Belgique devait appartenir au roi de France, parce qu'elle est habitée par la même race que la France, et parce qu'elle parle la langue française. En 1666, une telle théorie n'entrait encore dans l'esprit de personne, les universités allemandes n'ayant pas encore créé une ethnographie à l'usage des ambitieux ${ }^{87}$.

A ce jugement de Fustel de Coulanges fait écho ce compte rendu de P. Meyer :

84. On voit bien que dans ce cas les notions d'espace (comme support de discontinuités) et de temps (comme passé) sont des déterminations sociales et non des données immédiates de la conscience linguistique.

85. Que les linguistes allemands rattachent à l'influence du francisque, preuve supplémentaire de l'importance des langues germaniques dans la constitution de la langue française.

86. La Revue celtique a permis de désamorcer, sur le marché intellectuel, une revendication bretonne assimilée au catholicisme, au royalisme, en un mot, au passé.

87. Fustel de Coulanges, Questions contemporaines . la politique d'envahissement : Louvois et M. de Bismarck - (Hachette, Paris, 1919). Article paru en janvier 1871 dans la Revue des Deux Mondes. 
Il faut d'ailleurs s'abstenir de considérer la langue comme étant le signe de la nationalité. Elle peut seulement le devenir. L'idée que la patrie allemande, par exemple, s'étend partout où résonne la langue allemande, peut être à sa place dans une chanson, et étant, par sa simplicité même, à la portée des intelligences les plus ordinaires, a fait fortune, mais elle est à tout instant démentie par les faits. Les Wallons belges et les Genevois parlent français et ne sont ni ne veulent être français. Les Alsaciens étaient d'excellents Français tout en parlant allemand, et ne demandent qu'à le redevenir [...] Il faut étudier ces questions en elles-mêmes et sans préoccupation politique ${ }^{88}$.

Non seulement les linguistes français ne font pas de la langue un critère d'identification nationale, mais ils sont rejoints dans cette opinion par les défenseurs les mieux reconnus de l'occitan, comme F. Mistral (qui refuse toute idée d'un fédéralisme latin ou d'un séparatisme provençal) ou du breton comme Théodore Hersart de la Villemarqué.

La même idée pouvait apparaître comme interprétation du " darwinisme social ", d'autant plus aisément que son propagateur premier était un des plus célèbres linguistes, Max Muller. Il écrit : (de ce que les mêmes formes de langage) " se sont conservées chez tous les peuples de la famille aryenne, il suit qu'avant que les ancêtres des Hindous et des Perses se portassent vers le sud de l'Asie, et avant que les migrations asiatiques allassent former en Europe les colonies grecque, romaine, celtique, teutonique et slave, il existait une petite tribu aryenne, établie probablement dans la région la plus élevée de l'Asie centrale, parlant un langage qui n'était encore ni le sanscrit, ni le grec, ni l'allemand, mais qui contenait en germe les éléments dialectiques de toutes ces langues ${ }^{89}$ ". Pour comprendre quelle fut la réaction des linguistes français, il semble nécessaire d'étudier ceux qui se revendiquèrent particulièrement du darwinisme sur le marché de la linguistique. Deux noms y suffisent : Arsène Darmesteter et Abel Hovelacque.

A. Hovelacque (1843-1896) a suivi des études de linguistique notamment de grammaire comparée des langues indo-européennes : il est l'auteur d'une Grammaire de la langue zende (1869) - et d'anthropologie. Disciple de Broca et membre actif de sa "Société d'Anthropologie" (en 1890, il devient président de la Société d'Anthropologie et directeur de l'École de Broca), il a souci de mener de front recherche linguistique et enquête ethnographique qu'il répugne à séparer ${ }^{90}$. Il est aussi un homme politique, un républicain : le conseil municipal parisien le porte à sa tête en 1889 et il est élu député à Paris en 1893. Si Hovelacque reconnaît qu'il existe des races très distinctes, il se refuse à en trouver de pures en Europe : "Il est bien entendu que nous ne parlons ici que des individus parlant la langue basque et que la question spéciale de la

88. Paul Meyer, compte rendu du tiré à part de l'article de Henri Gaidoz paru dans les Annales de l'École libre des Sciences politiques: "Les vallées françaises du Piémont ", Romania, octobre 1887.

89. Max Müller, Lectures, $1^{\text {re }}$ série (1861). Ces Lectures on the Science of Language seront réfutées trente ans plus tard par les darwiniens (cf. I. Taylor, L'origine des Aryens et l'homme préhistorique, L. Battaille et Cie, Paris, 1895).

90. Cf. ses ouvrages : Études de linguistique et d'ethnographie (1878); Mélanges de linguistique et d'anthropologie (1880). 
race reste en dehors de cette statistique. Nous savons, en effet, grâce aux excellents travaux de $M$. Broca, qu'il y a Basques et Basques; que les Basques espagnols, par exemple, sont de sang bien moins mélangé que les Basques français ${ }^{91}$." Divergeant entièrement de Max Muller, Hovelacque affirme : "On commence aujourd'hui seulement à ne plus parler d'une race aryenne. On commence enfin à reconnaître qu'il y a bien une famille linguistique aryenne (langues de l'Inde du Nord, persan, grec, langues romanes, germaniques, slaves, lettiques), qu'il existe bien des langues aryennes, mais que l'on ne saurait parler en aucune façon d'une race aryenne. Nous rencontrons encore çà et là quelques attardés, mais en somme, sur cette question la lumière est faite et bien faite ${ }^{92}$. D Du point de vue des anthropologues, race et langue sont deux notions qui ne sauraient se recouvrir alors même que les linguistes affirment qu'il faut distinguer nation et langue.

Mieux introduit sur le marché de la linguistique, Arsène Darmesteter est beaucoup plus concerné par la définition raciale de l'indo-européen ${ }^{93}$. Comme Hovelacque a écrit La vie du langage ${ }^{94}$, Darmesteter a signifié son appartenance darwinienne en signant La Vie des mots (1887). Il est l'élève de Michel Bréal et de Gaston Paris qui l'a choisi comme répétiteur pour les langues romanes à l'École pratique des Hautes Études avant de lui offrir la chaire, créée à son intention, de "langue et littérature du Moyen Age " à la Sorbonne (1881). A. Darmesteter ne s'intéresse pas à l'étude des races; conformément à son approche " culturelle " du judaïsme, s'il assigne la linguistique aux sciences naturelles - et la littérature aux sciences historiques -, il fait de la rencontre des langues en France au Moyen Age l'anticipation d'une volonté et la découverte d'une identité. A propos du travail sur les gloses de Rashi ${ }^{95}$, James Darmesteter y voit, dans les Reliques scientifiques ${ }^{96}$, la conjonction du judaïsme et de la tradition philosophique et révolutionnaire française :

Qu'auraient dit les jongleurs et les clercs de Roland et de l'Alexis si on leur avait dit qu'un jour la langue de leurs chansons guerrières et de leurs pieuses homélies aurait besoin pour revenir au plein jour de l'aide du Ghetto et que le son vivant de leur parole serait rendu à la postérité par le grimoire anathémisé d'une race proscrite? Ame profondément éprise et du passé de sa race et de celui de sa patrie, Arsène mettait dans cette tâche comme un sentiment de double piété filiale : cette réconciliation que la philosophie et la Révolution ont faite entre les fils des persécuteurs et ceux des proscrits, il se sentait appelé à la refaire symboliquement dans le passé, et par la philologie il retrouvait l'âme commune des deux races ${ }^{97}$.

91. La linguistique, p. 148.

92. Hovelacque (avec J. Vinson), Études de linguistique et d'ethnographie (Paris, Reinwald, 1878, p. 271-272).

93. D'ores et déjà en Allemagne, l'appartenance à la communauté juive avait produit des effets (interdiction d'enseigner dans les universités de l'état par exemple) que la France connaîtra (affaire Dreyfus).

94. Hovelacque, op. cit. (p. 1 à 13) article paru dans La République française, le journal de Gambetta et de Joseph Reinach, le 20 juillet 1877 . Le " profil * de J. Reinach - dont un des frères est linguiste est proche de celui d'A. Darmesteter et de son frère James.

95. - Gloses et glossaires hébreux-français du Moyen Age *, Romania (1872).

96. Reliques scientifiques d'A. Darmesteter, publiées par J. Darmesteter (Cerf, Paris, 1890) 2 tomes.

97. Reliques..., p. Xvill (tome 1). 
Pour A. Darmesteter, le darwinisme régit la concurrence des espèces (les mots, les phénomènes, les langues entre elles et non les races) : les principes darwiniens de sélection, d'adaptation, de vie et de mort s'appliquent aux langues considérées comme des organismes vivants, en aucun cas à ceux qui les parlent ${ }^{98}$. Chercher pour quelle raison "honte " a remplacé "vergogne ", "entendre " "ouïr ", " renverser " "verser " ou $/ \mathrm{j} /$ le " 1 mouillé " ne suppose rien quant aux locuteurs, et quant à leur intervention dans la langue. Ainsi, A. Darmesteter participe à l'objectivation du fait linguistique construit comme élément d'un système indépendant des locuteurs. La logique de la structure et du changement linguistiques est à rechercher dans la langue seule.

Dans cette fraction du champ intellectuel dont la "Société de l'Enseignement supérieur " représente la quintessence, réfuter toute réflexion sur les civilisations occidentales conduite en terme de race devient un topique comme en témoigne Fustel de Coulanges : "Toute science chez eux (les Allemands) fut une arme contre la France. Ils inventèrent l'insoutenable théorie des races latines pour donner à leur ambition dynastique les faux dehors d'une querelle de races. Ils firent servir la philologie et l'ethnologie à démontrer que nos provinces les plus françaises étaient leur propriété légitime [... $]^{99}$." La "romanité ", système de traits culturels spécifique des peuples parlant les langues romanes s'oppose à la "race latine " des idéologues allemands et constitue une donnée du vocabulaire sociopolitique. "Roumanie " remplace "Moldavie-Valachie ", "Amérique latine " "colonies espagnoles et portugaises d'Amérique ", et, en France, avec l'approbation du mouvement provençal, la "latinité" devient le fondement obligé de toute définition d'identité nationale ${ }^{100}$.

Pourtant "deutschtum " et " romanité " (ou "latinité ") ne sont pas symétriques. Lorsque Gaston Paris écrit, en ouverture de la revue qu'il vient de fonder, son article sur " Roman " 101 , même s'il oppose constamment roman à germain, il n'assimile jamais à des races au sens strict les peuples. Comme Littré, il pense en termes de "nations " 102 , de peuple rassemblé, à peu près dans les termes qu'utilise Renan dans sa Nouvelle lettre $\dot{a}$ M. Strauss (1871):

L'individualité de chaque nation est constituée sans doute par la race, la langue, l'histoire, la religion, mais aussi par quelque chose de beaucoup plus tangible, par le consentement actuel, par la volonté qu'ont les différentes provinces d'un état de vivre ensemble [...]. La division trop accusée de l'humanité en races, outre qu'elle repose sur une erreur scientifique, très peu de pays possédant une race vraiment pure, ne peut mener qu'à des guerres d'extermination [...]. Vous avez levé dans le monde le drapeau de

98. Rompant avec les ambiguïtés de la théorie de Schleicher qui ne sépare pas langue et locuteur. 99. Op. cit., p. 69-70. Promu directeur de l'E.N.S., Fustel de Coulanges demanda à A. Darmesteter d'y faire un cours de romanistique. Sur cette question, voir aussi " Le langage et les nationalités " de M. Bréal dans la Revue des Deux Mondes (1-XII-1891).

100. Bien qu'une part de la défaite de 1870 soit imputée aux études classiques; cf. Raoul Frary, La question du latin (1871) et E. de Goncourt, Journal (en date du ler novembre 1870).

101. . Romani, Romania, lingua romana, romancium $*$, Romania (janvier 1872).

102. Littré : - Nation : réunion d'hommes habitant un même territoire, soumis ou non à un même gouvernement, ayant depuis longtemps des intérêts assez communs pour qu'on les regarde comme appartenant à la même race. " D'après Nicolet, op. cit., p. 400. 
la politique ethnographique et archéologique en place de la politique libérale; cette politique vous sera fatale ${ }^{103}$.

Voici les conclusions de l'article de Gaston Paris:

La Romania forme-t-elle vraiment un domaine intellectuel et moral, ou n'est-elle constituée que par l'origine commune des langues romanes? En d'autres termes, existe-t-il entre les peuples qui parlent aujourd'hui des dialectes latins des liens réels, autres que ceux de l'unité de langage, lesquels peuvent en somme être considérés comme purement fortuits et sont actuellement fort relâchés? [...] La Romania, ou I'union des nations romanes, n'a pas pour base une communauté de race. Quand on parle des races latines on emploie une expression qui manque absolument de justesse : il n'y a pas de races latines. La langue et la civilisation romaines ont été adoptées, plus ou moins volontairement, par les races les plus diverses, Ligures, Ibères, Celtes, Illyriens, etc ${ }^{104}$. C'est donc sur le sacrifice de la nationalité propre et originelle que repose l'unité des peuples romans; elle a pour base un principe tout différent de celui qui constitue l'unité germanique ou slave [...]. Dans le sein de cette association, les peuples romans forment un groupe plus étroitement uni, auquel s'opposent, tenant à l'ensemble par un lien de plus en plus lâche, les deux grandes nations des Germains et des Slaves. Chez ces peuples, la nationalité est exclusivement le produit du sang; la Romania au contraire est un produit tout historique. Son rôle paraît donc être, en face des sociétés qui ne sont que des tribus agrandies, de représenter la fusion des races par la civilisation. A ce principe, qui ne repose que sur une base physiologique, s'oppose heureusement celui qui fonde l'existence et l'indépendance des peuples sur l'histoire, la communauté des intérêts ${ }^{105}$ et la participation à une même culture. Il oppose le libre choix et l'adhésion qui provient de la reconnaissance des mêmes principes à la fatalité de la race; il est éminemment progressif et civilisateur, tandis que l'autre sera toujours par son essence conservateur et même exclusif. Ce n'est pas que le rationalisme pur, qu'on est habitué en France à introduire dans la politique, soit moins dangereux que l'esprit de race : le jeu opposé du principe de tradition et du principe de progrès est la condition de tout développement régulier. Mais le lien qui unit entre elles les nations romanes est précisément, par sa nature, à la fois traditionnel et rationnel. Héritières de Rome, elles doivent conserver de son esprit ce qui est le plus utile à l'humanité, la tendance vers une civilisation commune, équitable et éclairée [...]. La fraternité des diverses nations romanes est sensible dans l'histoire de leurs littératures autant que dans celle de leurs langues [...]. Cette individualité ${ }^{106}$ a même été assez marquée pour que le sentiment de la communauté ait pu presque s'effacer : il doit trouver une reviviscence durable dans l'étude des langues et des littératures romanes, à laquelle nous consacrons ce recueil. Nous ne poursuivons, cela va sans dire, d'autre intérêt que celui de la science : les faits seuls parleront; nous ne les choisirons ni ne les interpréterons avec aucune idée préconçue. Mais c'est précisément au point de vue purement scientifique que le rapprochement perpétuel entre les langues et les littératures des diverses nations qui composent la Romania est le plus utile et le plus fructueux $[\ldots]^{107}$."

103. Cité dans H. Peyre, Renan (Paris, 1969) et Nicolet, op. cit., p. 406.

104. Ênumération qui justifie le a gallo-roman au détriment de l'occitan.

105. Cette définition est, au mot près, celle que donne Littré.

106. Il s'agit de l'individualité prise par chacun des peuples romans en raison des contacts.

107. G. Paris, article cité et repris dans les Mélanges linguistiques publiés en quatre fascicules par la * Société amicale Gaston Paris *, à Paris, en 1905 (fascicule 1, p. 28-31). 
Par patriotisme, mais au nom de la science, les comparatistes entreprennent de réfuter les propositions allemandes sur linfluence de la culture germanique ou sa supériorité vis-à-vis des langues et littératures romanes. La thèse d'A. Darmesteter, pour son diplôme de sortie de l'E.P.H.E. (1872) est idéaltypique. Son frère le souligne : "Il avait pris un sujet exclusivement français : de la formation des mots composés en français. C'était un sujet qui semblait bien maigre : car c'était un des lieux communs de la philosophie courante que les langues romanes ne connaissent pas la composition qui est un des privilèges et une des supériorités des langues germaniques. Le livre d'Arsène fit justice de ce cliché d'une façon définitive, avec une richesse de faits et une puissance d'analyse qui ne laissait plus place au doute ${ }^{108}$. "Sa thèse mineure concerne cette part de la littérature médiévale française dont les critiques allemands situaient les sources dans leur propre pays en prenant exemple sur le Roman de Renart dont l'onomastique est germanique. Il étudie l'origine de Floovant dont il retrouve le thème dans la littérature byzantine à laquelle les littératures européennes l'auraient emprunté.

Mais c'est en fondant la partie scientifique du marché de la linguistique - dont ils obtiennent l'intégration à l'université - que les comparatistes ont contribué à la " revanche " intellectuelle attendue par les locuteurs français, à la formation de la doxa nationale et républicaine en gestation dans le champ politique et aux progrès de la romanistique et de l'orientalisme.

\section{Conclusion}

De 1865 à 1885 , la linguistique française a porté sa réflexion de la délimitation de l'objet linguistique aux limites de l'objet linguistique. A partir de 1865, la fraction comparatiste du marché de la linguistique a procédé à un travail continu de démarcation :

- démarcation entre le linguistique et le non-linguistique, c'est-à-dire entre la langue et les autres systèmes d'identité symbolique des locuteurs (race, nationalité, religion...) ${ }^{109}$, entre le langage et ses formes de représentation (écriture, orthographe, littérature) ${ }^{110}$, entre le plan de l'expression et le plan du contenu '11.

- démarcation entre les savants qui désenchantent les études littéraires et les mondains qui conservent leur attachement à une vision romantique de l'histoire, de la langue et de la littérature ${ }^{112}$.

108. A. Darmesteter, Reliques scientifiques (librairie L. Cerf, Paris, 1890). Préface de James Darmesteter, tome I, p. XX.

109. Démarcation que refusent les provençalistes mais qui contribue à l'émergence de l'ethnologie, des études de folklore, de l'anthropologie...

110. Démarcation refusée par les mondains, puristes ou grammairiens, et qui distingue linguistique et philologie.

111. Pour reprendre les concepts de Hjelmslev, Bréal avait prononcé une suite de conférences sur - les idées latentes dans le langage - en 1868 mais il abandonna ce thème pendant quinze ans.

112. Dans Le crime de Sylvestre Bonnard, Anatole. France exploite cette situation à des fins romanesques: " M. de Lessay, apprenant que je sortais de l'École des Chartes, me jugea digne de collaborer à son atlas historique. Il s agissait de déterminer sur une suite de cartes ce que le vieillard philosophe nommait les vicissitudes des empires depuis Noë jusqu'à Charlemagne. M. de Lessay avait emmagasiné 
- démarcation sociale entre les jeunes chercheurs, laïques et républicains, dont le patriotisme, universalisé dans la figure de la science, refuse le morcellement de la nation et son infériorité face à l'Allemagne, et les universitaires traditionnels, plus âgés, attachés aux études classiques, catholiques ${ }^{113}$ et conservateurs, tenants de la supériorité de la littérature sur la linguistique et du "goût "français sur le pédantisme germanique. Le marché de la linguistique se divise non seulement sur la conception de la langue mais aussi sur la définition du patriotisme, les comparatistes reprenant les théories allemandes contre l'Allemagne, les mondains refusant tout ce qui est allemand, fût-ce de la science.

Jusqu'aux années 1880 , romanistes et orientalistes se déprennent du marché linguistique et assurent l'autonomie de leur discipline en disposant les marques d'une pratique ésotérique de la connaissance : système d'écriture spécifique, corpus de référence, appareil de règles et de lois, méthodes d'interprétation et fermeture des séminaires et des cours, diplômes particuliers, recrutement indépendant.

Vers 1885 , le concept de langue, tel qu'il a été construit dans et pour le travail de démarcation scientifique et sociale, semble restrictif en regard des recherches en cours. La séparation avec les mondains et les universitaires de la génération précédente est acquise puisque les savants se sont progressivement substitués à leurs concurrents dans l'enseignement supérieur. Le contrôle de l'université est confié par étapes aux partisans de la Société de l'Enseignement supérieur ${ }^{114}$. Les hauts fonctionnaires de l'Instruction publique ont appuyé l'investissement des orientalistes et romanistes dans l'enseignement supérieur qui leur assure, en une vingtaine d'années, un monopole sur la linguistique. En identifiant leur stratégie à un combat pour la science (et pour la science au service de la patrie), les comparatistes ont pu se dispenser des interrogations sur la légitimité sociale de leur action dans la mesure où elle les dépassait en tant que personnes. Ils attribuent leurs succès à la reconnaissance de la vérité plus qu'aux soutiens qu'ils ont reçus ou constitués (les sociétés et les revues par exemple).

Quant au concept de langue construit entre 1865 et 1880 pour disqualifier leurs concurrents, les linguistes sont tenus de le relativiser en trois points :

1) la transcription: le recours à la phonétique expérimentale bouleverse les notations phonétiques traditionnelles fondées sur la perception. En

dans sa tête toutes les erreurs du Xville siècle en matière d'antiquités. J'étais, en histoire, de l'école des novateurs et dans un âge où l'on ne sait guère feindre. La façon dont le vieillard comprenait ou plutôt ne comprenait pas les temps barbares, son obstination à voir dans la haute Antiquité des princes ambitieux, des prélats hypocrites et cupides, des citoyens vertueux, des poètes philosophes et autres personnages, qui n'ont jamais existé que dans les romans de Marmontel, me rendait horriblement malheureux et $m$ 'inspira d'abord toutes sortes d'objections fort rationnelles sans doute mais parfaitement inutiles et quelquefois dangereuses * (Calmann-Lévy, 1924, p. 167). A. France (né en 1844) avait a fréquenté . l'École des Chartes à la fin des années 1860.

113. Il est significatif que les deux plus grands linguistes français avant 1870, Renan et Littré, soient présentés par l'église catholique comme de redoutables adversaires et qu'ils soient obligés de poursuivre leurs recherches en marge des facultés.

114. A l'initiative de Louis Liard, directeur de l'enseignement supérieur au ministère de l'Instruction publique de 1885 à 1902 , Goblet promeut une réforme des facultés par décrets. 
s'intéressant aux phénomènes de production, Rousselot ${ }^{115}$ pense les systèmes phonétiques comme autant de cas particuliers dans l'ensemble des possibilités articulatoires;

2) la dialectologie: la nécessité de préparer le travail d'enquête par la formation linguistique - et plus particulièrement phonétique - du collecteur et de dresser un atlas pour obtenir une représentation scientifique des patois approfondit l'écart entre " professionnels " et " dilettantes " sur le marché de la linguistique mais réduit la distance entre langue et dialecte d'une différence d'essence à une différence de degrée ${ }^{116}$;

3) la signification : la question du sens linguistique, principal obstacle à l'objectivation des faits de langue, fait retour dans les travaux d'A. Darmesteter (confronté à une ouvre lexicographique mettant en jeu un classement chronologique et logique du sens) ${ }^{117}$ et de M. Bréal ${ }^{118}$.

Ces trois questions ${ }^{119}$, qui apparaissent simultanément entre 1883 et 1886, au moment où la configuration institutionnelle du marché de la linguistique paraît achevée dans ses postes et dans leurs détenteurs, sont au principe du retour opéré par les comparatistes vers le marché linguistique. Forts de leur autorité scientifique et universitaire, les linguistes inaugurent une pratique exotérique de leur discipline dont ils tentent d'imposer l'autorité à l'ensemble des locuteurs (et non plus seulement à leurs concurrents). Ce retour au marché linguistique, lié aux progrès sociaux et scientifiques de la romanistique française, concerne d'abord les programmes scolaires de l'enseignement secondaire ${ }^{120}$ puis la question de la réforme orthographique ${ }^{121}$.

Alors que la construction du marché de la linguistique autour de l'idée de science avait permis aux comparatistes de s'assurer une autorité disproportionnée par rapport à leur capital universitaire, le retour vers le marché linguistique sera un échec, dû en partie à la transformation du marché des biens symboliques, en liaison, probablement, avec les partages nouveaux opérés par l'affaire Dreyfus. Le rôle de la linguistique devient moins crucial et le principe de démarcation n'est plus attendu de cette discipline mais des sciences nouvelles qui cherchent les fondements d'une cohésion menacée par les affrontements politiques : il semble que ce soit un des rôles de la sociologie durkheimienne ${ }^{122}$. Ce sera l'objet d'un article ultérieur ${ }^{123}$.

115. Les premiers travaux de Rousselot en phonétique se situeraient vers 1885 .

116. Les cours de dialectologie de Gilliéron à l'E.P.H.E. commencent en 1883 .

117. La vie des mots paraît en 1886.

118. Cf. notamment sa critique du livre de Darmesteter : * L'histoire des mots * (1887) reproduit dans l'Essai de sémantique (1897).

119. Auxquelles il faudrait ajouter la méthodologie comparatiste rediscutée sous l'influence des néo-grammairiens allemands (1876 sq.) et de Saussure (qui enseigne à l'E.P.H.E. de 1881 à 1891 ).

120. Des cours d'histoire de la langue française sont inclus dans les programmes des lycées en 1880 , accrus en $1885 \ldots$ et abandonnés en 1890 .

121. En 1887, Paul Passy fonde une association pour la réforme de l'orthographe que soutiennent G. Paris, P. Meyer, L. Clédat, M. Bréal, L. Havet... et dont les vicissitudes diviseront le marché intellectuel jusqu'à 1910 .

122. Cf. notamment les articles de P. Birnbaum, J.-C. Filloux et V. Karady dans le numéro d'avriljuin 1976 de la Revue française de sociologie intitulé * A propos de Durkheim *.

123. G. Bergounioux, "Le marché de la linguistique en France (1885-1914) *. A paraître. 


\begin{tabular}{|c|c|c|c|}
\hline Établissement & 1870 & 1878 & 1884 \\
\hline Collège de $\mathrm{F}$. & $\begin{array}{l}\text { BRÉAL M. }{ }^{\bullet} \\
\text { PARIS Gaston } \triangle \\
\text { QUINET E. }\end{array}$ & $\begin{array}{l}\text { BRÉAL • } \\
\text { PARIS } \triangle \\
\text { MEYER }^{\triangle}\end{array}$ & $\begin{array}{l}\text { BRÉAL • } \\
\text { PARIS } \triangle \\
\text { MEYER } \triangle \\
\text { D'ARBOIS DE JUBAINVILLE } \triangle\end{array}$ \\
\hline E.P.H.E. & $\begin{array}{l}\text { PARIS } \triangle \\
\text { BRACHET } \triangle\end{array}$ & $\begin{array}{l}\text { PARIS } \triangle \\
\text { DARMESTETER } \square \\
\text { GAIDOZ }\end{array}$ & $\begin{array}{l}\text { PARIS } \triangle \\
\text { GILLIERON } \triangle \\
\text { GAIDOZ } \\
\text { LONGNON } \square\end{array}$ \\
\hline Chartes & $\begin{array}{l}\text { MONTAIGLON }{ }^{\Delta} \\
\text { MEYER }^{\Delta} \\
\text { GAUTIER }^{\Delta}\end{array}$ & $\begin{array}{l}\text { MONTAIGLON } \triangle \\
\text { MEYER }^{\Delta} \\
\text { GAUTIER }^{\Delta}\end{array}$ & $\begin{array}{l}\text { MONTAIGLON } \Delta \\
\text { MEYER }^{\Delta} \\
\text { GAUTIER }^{\Delta}\end{array}$ \\
\hline E.N.S. & THUROT & THUROT & PETIT DE JULLEVILLE • \\
\hline Sorbonne & SAINT-MARC-GIRARDIN & $\begin{array}{l}\text { LEENIENT } \bullet \\
\text { DARMESTETER A. }\end{array}$ & $\begin{array}{l}\text { LÉNIENT } \bullet \\
\text { DARMESTETER A. }\end{array}$ \\
\hline Montpellier & & $\begin{array}{l}\text { BOUCHERIE } \\
\text { CHABANEAU }\end{array}$ & CHABANEAU \\
\hline Lyon & & CLÉDAT ㅁ & CLÉDAT $\square$ \\
\hline Caen & . & GASTE • & GASTE • \\
\hline Rennes & & & LOTH \\
\hline Genève & & RITTER & RITTER \\
\hline Aix-en-P. & & & $\begin{array}{l}\text { JORET } \square \\
\text { CONSTANS }\end{array}$ \\
\hline Bordeaux & & & LUCHAIRE व \\
\hline Toulouse & & & THOMAS $\square$ \\
\hline Dijon & & PETIT DE JULLEVILLE • & \\
\hline École Broca & & HOVELACQUE & HOVELACQUE \\
\hline $\begin{array}{l}\text { Fac. Catho. } \\
\text { (Lyon) }\end{array}$ & & DEVAUX & DEVAUX \\
\hline $\begin{array}{l}\text { Fac. Libre } \\
\text { (Toulouse) }\end{array}$ & & & COUTURE \\
\hline $\begin{array}{l}\text { École Sup. } \\
\text { (Alger) }\end{array}$ & & & MOREL-FATIO $\triangle$ \\
\hline
\end{tabular}

